\title{
Role of a1 and a 2 chains of type IV collagen in early fibrotic lesions of idiopathic interstitial pneumonias and migration of lung fibroblasts
}

\author{
Hirokazu Urushiyama', Yasuhiro Terasaki ${ }^{1}$, Shinya Nagasaka', Mika Terasaki ${ }^{1}$, Shinobu Kunugi ${ }^{1}$, Takahide Nagase², \\ Yuh Fukuda ${ }^{1}$ and Akira Shimizu'
}

Early fibrotic lesions are thought to be the initial findings of fibrogenesis in idiopathic interstitial pneumonias, but little is known about their properties. Type IV collagen comprises six gene products, a1-a6, and although it is known as a major basement membrane component, its abnormal deposition is seen in fibrotic lesions of certain organs. We studied the expression of type I and III collagen and all a chains of type IV collagen in lung specimens from patients with usual interstitial pneumonia (UIP) or organizing pneumonia (OP) via immunohistochemistry. With cultured lung fibroblasts, we analyzed the expression and function of all a chains of type IV collagen via immunohistochemistry, western blotting, realtime quantitative PCR, and a Boyden chamber migration assay after the knockdown of $a 1$ and a2 chains. Although we observed type I and III collagens in early fibrotic lesions of both UIP and OP, we found type IV collagen, especially a1 and a2 chains, in early fibrotic lesions of UIP but not OP. Fibroblasts enhanced the expression of $a 1$ and $a 2$ chains of type IV collagen after transforming growth factor- $\beta 1$ stimulation. Small interfering RNA against $\alpha 1$ and $\alpha 2$ chains increased fibroblast migration, with upregulated phosphorylation of focal adhesion kinase (FAK), and adding medium containing fibroblast-produced $\mathrm{a} 1$ and $\mathrm{a} 2$ chains reduced the increased levels of fibroblast migration and phosphorylation of FAK. Fibroblasts in OP were positive for phosphorylated FAK but fibroblasts in UIP were not. These results suggest that fibroblasts in UIP with type IV collagen deposition, especially a1 and a2 chains, have less ability to migrate from early fibrotic lesions than fibroblasts in OP without type IV collagen deposition. Thus, type IV collagen deposition in early fibrotic lesions of UIP may be implicated in refractory pathophysiology including migration of lesion fibroblasts via a FAK pathway. Laboratory Investigation (2015) 95, 872-885; doi:10.1038/labinvest.2015.66; published online 25 May 2015

Idiopathic interstitial pneumonias (IIPs) make up a heterogeneous group of nonneoplastic disorders resulting from lung parenchyma damage of unknown origin. IIPs have various patterns of inflammation and fibrosis, and clinical diagnosis of IIPs is based on clinical-radiologic-pathologic features. Of IIPs, idiopathic pulmonary fibrosis (IPF)/usual interstitial pneumonia (UIP) is said to be in the group having a poor prognosis because of poor responses to treatment, whereas cryptogenic organizing pneumonia (COP)/organizing pneumonia (OP) is in the group with a good prognosis because of a better response to treatment. ${ }^{1,2}$

IIPs have early fibrotic lesions that contain small aggregates of myofibroblasts and fibroblasts with extracellular matrix. These early fibrotic lesions, including fibroblastic foci in UIP and intraluminal buds in $\mathrm{OP}$, are thought to be initial findings of lung fibrogenesis. These early fibrotic lesions differ not only in tissue morphology but also in properties affecting lung fibrogenesis. For example, we previously showed that deposition of matrix metalloproteinase-2, which functions in extracellular matrix degradation, is greater in intraluminal buds of OP, whereas deposition of tissue inhibitors of metalloproteinase-2 is greater in fibroblastic foci of UIP. ${ }^{3}$ Transforming growth factor (TGF)- $\beta 1$, which is involved in fibrosis, is clearly present in fibroblastic foci of UIP. ${ }^{4}$

The extracellular matrix provides structural support, but studies have also provided intriguing evidence that a wealth of biochemical information is contained within its molecular structure. ${ }^{5,6}$ Moreover, several basement membrane (BM) components, and even fragments of them, were found to regulate cell function. For example, endostatin, ${ }^{7}$ a $20-\mathrm{kDa}$

\footnotetext{
${ }^{1}$ Department of Analytic Human Pathology, Graduate School of Medicine, Nippon Medical School, Tokyo, Japan and ${ }^{2}$ Department of Respiratory Medicine, Graduate School of Medicine, The University of Tokyo, Tokyo, Japan

Correspondence: Dr Y Terasaki MD, PhD, Department of Analytic Human Pathology, Graduate School of Medicine, Nippon Medical School, 1-1-5 Sendagi, Bunkyo-ku, Tokyo 113-8602, Japan.

E-mail: terasaki@nms.ac.jp
}

Received 15 April 2014; revised 6 March 2015; accepted 7 April 2015 
C-terminal fragment of type XVIII collagen that is a component of vascular and epithelial BMs, inhibits endothelial cell migration ${ }^{8}$ and angiogenesis. Type IV collagen is also known as an important structural component of the $\mathrm{BM}^{9}$ and comprises six genetically distinct products of the COL4A1COL4A6 genes $-\alpha 1-\alpha 6$ chains of type IV collagen, or $\alpha 1$ (IV) to $\alpha 6$ (IV) chains. ${ }^{10}$ In type IV collagen, arresten ${ }^{11}$ and canstatin, ${ }^{12}$ which are parts of the C-terminal globular noncollagenous (NC1) domains of $\alpha 1$ (IV) and $\alpha 2$ (IV) chains, respectively, inhibit endothelial cell migration. In clinical situations, serum levels of type IV collagen in liver fibrosis and urine levels of type IV collagen in diabetic nephropathy reportedly correlate with the degree of liver and kidney fibrosis, respectively. ${ }^{13,14}$ The serum concentrations of type IV collagen also increased in patients with IPF. ${ }^{15}$ However, little is known of the expression and role of type IV collagen, especially in early fibrotic lesions of IIPs. To investigate the precise expression and role of type IV collagen in IIPs, we studied the expression, localization, and possible function of each type IV collagen $\alpha$ chain by using human lung tissue sections diagnosed as UIP and OP, as well as cultured human lung fibroblasts.

\section{MATERIALS AND METHODS Patients}

Lung specimens were obtained by performing surgical lung biopsies (14 UIP and 11 OP cases) at the Nippon Medical School Hospital from 1992 to 2011. All specimens were diagnosed by using the American Thoracic Society/European Respiratory Society International Multidisciplinary Consensus Classification of the Idiopathic Interstitial Pneumonias. ${ }^{1,2}$ The study protocol was approved by the Human Ethics Review Committee of Nippon Medical School. Written informed consent was obtained from patients. Table 1 shows the demographic characteristics and pulmonary function data for patients at the time of biopsy.

\section{Analysis for Early Fibrotic Lesions of UIP and OP}

For patients with UIP, surgical lung biopsy specimens were obtained from the upper and lower lobes in 10 patients, from three lobes in one patient, and from the lower lobes in three patients. For patients with OP, specimens were obtained from the two diseased lung lobes in five patients and from the single diseased lobe in six patients. Two authors (HU, YT), without knowing the clinical information, reviewed all fibroblastic foci in UIP and intraluminal buds in OP on all available slides from surgical lung biopsies. The average observed numbers of early fibrotic lesions were 7.04 per lung lobe for patients with UIP and 48.9 per lung lobe for patients with OP.

\section{Cell Culture}

Human diploid fetal lung fibroblasts of the cell line TIG-1-20 (ref. 16) were provided by the Cell Resource Center for Biomedical Research, Institute of Development, Aging and
Table 1 Baseline characteristics of patients with UIP and OP at the time of biopsy

\begin{tabular}{lcc}
\hline & UIP $(n=14)$ & OP $(n=11)$ \\
\hline Age, years (range) & $62.9(49-76)$ & $58.9(43-71)$ \\
Sex, male/female & $12 / 2$ & $5 / 6$ \\
Smoking status, ever/never & $12 / 2$ & $6 / 5$ \\
Interval between onset and biopsy, & $18.7 \pm 16.9$ & $3.41 \pm 1.39$ \\
months & & \\
FVC, liters & $2.52 \pm 0.98$ & $3.01 \pm 0.49$ \\
FVC predicted, \% & $77.7 \pm 27.5$ & $102.3 \pm 11.0$ \\
DLCO predicted, \% (total number & $42.8 \pm 11.0(n=5)$ & $68.4 \pm 8.74(n=3)$ \\
with data) & & \\
\hline
\end{tabular}

Data are expressed as means \pm s.d.

Cancer, Tohoku University, and were purchased from the Health Science Research Resources Bank. TIG-1-20 cells were cultured in Dulbecco's modified Eagle's medium (DMEM; Wako Pure Chemical Industries, Osaka, Japan) supplemented with $10 \%$ fetal bovine serum (FBS), $50 \mu \mathrm{g} / \mathrm{ml}$ streptomycin, and $50 \mathrm{U} / \mathrm{ml}$ penicillin at $37^{\circ} \mathrm{C}$ under humidified $5 \% \mathrm{CO}_{2} /$ $95 \%$ air.

\section{Immunohistochemistry}

Serial sections of deparaffinized, rehydrated lung tissues were washed with phosphate-buffered saline (PBS) and treated with $0.3 \% \mathrm{H}_{2} \mathrm{O}_{2}$ in methanol for $30 \mathrm{~min}$ to eliminate endogenous peroxidase activity. To unmask antigenic epitopes, sections to be used for type I, III, and IV collagen staining were treated with $1 \%$ pepsin in $0.01 \mathrm{~N} \mathrm{HCl}$ for $60 \mathrm{~min}$, sections to be used for analyses of $\alpha 1$ through $\alpha 6$ chains of type IV collagen were heated at $132{ }^{\circ} \mathrm{C}$ for $30 \mathrm{~min}$ with $0.01 \mathrm{M}$ citrate buffer $(\mathrm{pH}$ $3.3)$, sections to be used for $\alpha$-smooth muscle actin ( $\alpha$-SMA) studies were heated at $95^{\circ} \mathrm{C}$ for $45 \mathrm{~min}$ with $0.5 \%$ ImmunoSaver (Nissin EM, Tokyo, Japan), and sections to be used for examinations of phosphorylated focal adhesion kinase (FAK; phospho Y397) were heated at $95^{\circ} \mathrm{C}$ for $45 \mathrm{~min}$ with $0.01 \mathrm{M}$ citrate buffer $(\mathrm{pH}$ 6.0). Sections were then incubated with appropriate dilutions of each primary antibody. Primary antibodies were goat polyclonal anti-type I collagen (1:300 dilution), anti-type III collagen (1:300), and anti-type IV collagen (1:300; SouthernBiotech Associates, Birmingham, AL, USA); rat monoclonal anti- $\alpha$ (IV) chains, H11, H22, H31, $\mathrm{H} 43, \mathrm{H} 52$, and H63, specific for $\alpha 1$ (IV), $\alpha 2$ (IV), $\alpha 3$ (IV), $\alpha 4$ (IV), $\alpha 5(\mathrm{IV})$, and $\alpha 6(\mathrm{IV})$ chains, respectively (1:100; a kind gift from Dr Sado); ${ }^{17}$ mouse monoclonal anti-human $\alpha$-SMA (1:300; Dako, Glostrup, Denmark); and rabbit polyclonal antiFAK-pY397(1:100; Abcam, Cambridge, MA, USA). Sections were then incubated with Histofine Simple Stain Kits (Nichirei Biosciences, Tokyo, Japan) as secondary antibodies with peroxidase for $30 \mathrm{~min}$. Peroxidase activity was detected with a solution of $3,3^{\prime}$-diaminobenzidine and $\mathrm{H}_{2} \mathrm{O}_{2}$, with the 
counterstain being Mayer's hematoxylin. The criteria indicating specific immunohistochemical features were as follows: strongly positive staining meant that early fibrotic lesions were clearly distinguishable from the surrounding area via immunohistochemical staining; weakly positive staining meant that early fibrotic lesions were not clearly distinguishable from the surrounding area but that positive results were present in early fibrotic lesions in high-power fields $(\times 400)$; and negative results meant that no positive staining results were present in early fibrotic lesions in high-power fields (Supplementary Figure 1).

\section{Western Blotting}

To examine the production of $\alpha$ (IV) chains in TIG-1-20 cells, cultured medium with FBS was replaced with serum-free DMEM on day 1 after passage. TIG-1-20 cells were then cultured with various concentrations of recombinant human TGF- $\beta 1$. After the indicated time of culture, the medium was collected and TIG-1-20 cells were homogenized in M-PER (Mammalian Protein Extraction Reagent; Thermo Scientific, Rockford, IL, USA) containing a protein-stabilizing cocktail (Halt Protease Inhibitor Cocktail; Thermo Scientific), a phosphatase inhibitor (Halt Phosphatase Inhibitor Cocktail; Thermo Scientific), $150 \mathrm{mM} \mathrm{NaCl}$, and $1 \mathrm{mM}$ EDTA. Homogenized samples were centrifuged (15000 r.p.m., 25 min), and the supernatant was called cell lysate. Then, $15 \mu$ l of SDS sample buffer with 2-mercaptoethanol for reduction was added to an equal amount of samples of cultured medium or lysates. These samples were boiled at $95^{\circ} \mathrm{C}$ for $5 \mathrm{~min}$, subjected to SDS-polyacrylamide gel electrophoresis with $10 \%$ acrylamide separating gel (e-PAGEL; ATTO, Tokyo, Japan), and transferred to polyvinylidene difluoride membranes (Invitrogen, Carlsbad, CA, USA) with an electroblot apparatus (Invitrogen). Membranes were incubated for $1 \mathrm{~h}$ at room temperature with protein-free T20 Tris-buffered saline blocking buffer (Thermo Scientific) and were then incubated at $4{ }^{\circ} \mathrm{C}$ overnight with antibodies against $\alpha 1$ (IV), $\alpha 2$ (IV), $\alpha 3$ (IV), $\alpha 4(\mathrm{IV}), \alpha 5(\mathrm{IV})$, and $\alpha 6$ (IV) chains at a dilution of $1: 300$, or antibodies against $\alpha$-SMA, and $\alpha$-tubulin (Epitomics, Burlingame, CA, USA) at a dilution of 1:3000. After the membranes were washed several times with Tris-buffered saline containing $0.1 \%$ Tween 20 , they were incubated with the appropriate horseradish peroxidase-conjugated second antibodies (Promega, Madison, WI, USA) for $60 \mathrm{~min}$, washed with Tris-buffered saline containing $0.1 \%$ Tween 20, and developed with SuperSignal West Femto Luminol/Enhancer solution (Thermo Scientific). Immunoreactivity on blots was detected with the LAS-4000 Luminescent Image Analyzer with CCD Camera (Fujifilm, Tokyo, Japan) and was quantified by using densitometry with Fuji Image Gauge software (version 4.0; Fujifilm). After the antibodies were stripped from the blotting membrane by $15 \mathrm{~min}$ of incubation in Restore PLUS Western Blot stripping buffer (Thermo Scientific), $\alpha$-tubulin immunoreactivity of the same membrane was quantified by using the same western blotting method. Production of each protein was determined as a ratio to the amount of $\alpha$-tubulin protein, and results were reported relative to culture without TGF- $\beta 1=1.0$.

\section{Real-Time Quantitative Reverse Transcription-PCR Amplification}

To study mRNA expression of $\alpha$ chains of type IV collagen in TIG-1-20 cells, cultured medium with FBS was replaced with serum-free medium on day 1 after passage. Cells were then cultured with various concentrations of recombinant human TGF- $\beta 1$ for the indicated time points. Total RNA was purified from the cells after various treatments by using the RNeasy Mini Kit (Qiagen Sciences, Germantown, MD, USA) according to the manufacturer's instructions. Reverse transcription was performed at $25^{\circ} \mathrm{C}$ for $10 \mathrm{~min}, 37^{\circ} \mathrm{C}$ for $120 \mathrm{~min}$, and $85^{\circ} \mathrm{C}$ for $4 \mathrm{~min}$. After reverse transcription, quantitative reverse transcription-PCR (RT-qPCR) amplifications were performed with TaqMan Universal PCR Master Mix (Applied Biosystems, Foster City, CA, USA) on the ABI PRISM 7900HT system (Applied Biosystems). Ready-to-use primer and probe sets (Assays-on-Demand gene expression: numbers

Figure 1 Type I, III, and IV collagens and a-smooth muscle actin (a-SMA) in normal control lung and early fibrotic lesions of usual interstitial pneumonia (UIP) and organizing pneumonia (OP). Representative hematoxylin-eosin-stained specimens of normal control lung (a), UIP (b)), and OP (c). Images revealed histologic features of UIP including architectural destruction, fibrosis with honeycombing, and scattered fibroblastic foci (b), and histologic features of OP including patchy lung involvement by loose polyps of connective tissue within alveolar air spaces (c). Insets show high-magnification views of the red rectangular areas: normal alveoli and interstitium around a pulmonary vessel (a), a fibroblastic focus (b), and an intraluminal polyp (c). In normal lung, type I (d) and type III (g) collagens were ubiquitous in the alveolar region (insets) and in the interstitium of all lung components (black stars), whereas type IV collagen (j) was present only in the basement membrane (BM) of the epithelium (black arrows), endothelium (white arrows), and smooth muscle cells (arrowheads), but not in the interstitium around a pulmonary vessel (white star). Insets show high-magnification views of normal alveoli in the red rectangular areas (d, $\mathbf{g}$ and $\mathbf{j}$ ). In idiopathic interstitial pneumonias (IIPs), deposits of type I collagen (e and $\mathbf{f}$, black stars) and type III collagen (h and $\mathbf{i}$, black stars) were clearly seen in early fibrotic lesions of UIP and OP as well as in the interstitium of normal lung (d and $\mathbf{g}$, black stars). However, type IV collagen was clearly deposited in early fibrotic lesions of UIP (k, black star) but not in early fibrotic lesions of OP (I, white star). Smooth muscle cells in normal lung ( $\mathbf{m}$, arrowheads) and fibroblasts in fibroblastic foci of UIP (n, black star), showed a-SMA-positive staining, whereas fibroblasts in intraluminal buds of OP (o, white star) had little or no a-SMA-positive staining. High-magnification views of fibroblastic foci in the red rectangular areas (k and $\mathbf{n}$, insets) show that type IV collagen deposited in a fine fibrillar pattern (black arrows) around a-SMA-positive fibroblasts (arrowheads), distinct from the BM structure in UIP, whereas high-magnification views of intraluminal buds in the red rectangular areas (I and $\mathbf{0}$, insets) show no type IV collagen deposits around $\mathbf{a}$-SMA-negative fibroblasts in OP. UIP images ( $\mathbf{b}, \mathbf{e}, \mathbf{h}, \mathbf{k}$ and $\mathbf{n}$ ) were obtained from the same patient, as were OP images (c, f, i, I and $\mathbf{o}$ ) and normal control lung images (a, d, $\mathbf{g}, \mathbf{j}$ and $\mathbf{m})$. Scale bars are $500 \mu \mathrm{m}(\mathbf{a}-\mathbf{c})$ and $100 \mu \mathrm{m}(\mathbf{d}-\mathbf{o})$. 
Hs00266237_ml, Hs01098873 m1, Hs01022542 m1, Hs01011885_ml, Hs00166712 m1, Hs00361494_ml, and glyceraldehyde-3-phosphate dehydrogenase (GAPDH; Applied Biosystems) were used to detect COL4A1, COL4A2, COLAA3, COL4A4, COL4A5, COL4A6, and GAPDH. Two microliters from the RT reaction was used in the quantitative PCR reactions (total volume of $25 \mu \mathrm{l}$ ). The thermal profile of RT-qPCR was $95^{\circ} \mathrm{C}$ for $10 \mathrm{~min}$ followed by 40 cycles that had the temperature protocol set at $95^{\circ} \mathrm{C}$ for $15 \mathrm{~s}$ and then at $60^{\circ} \mathrm{C}$ for $60 \mathrm{~s}$. Ratios of mRNA expressions
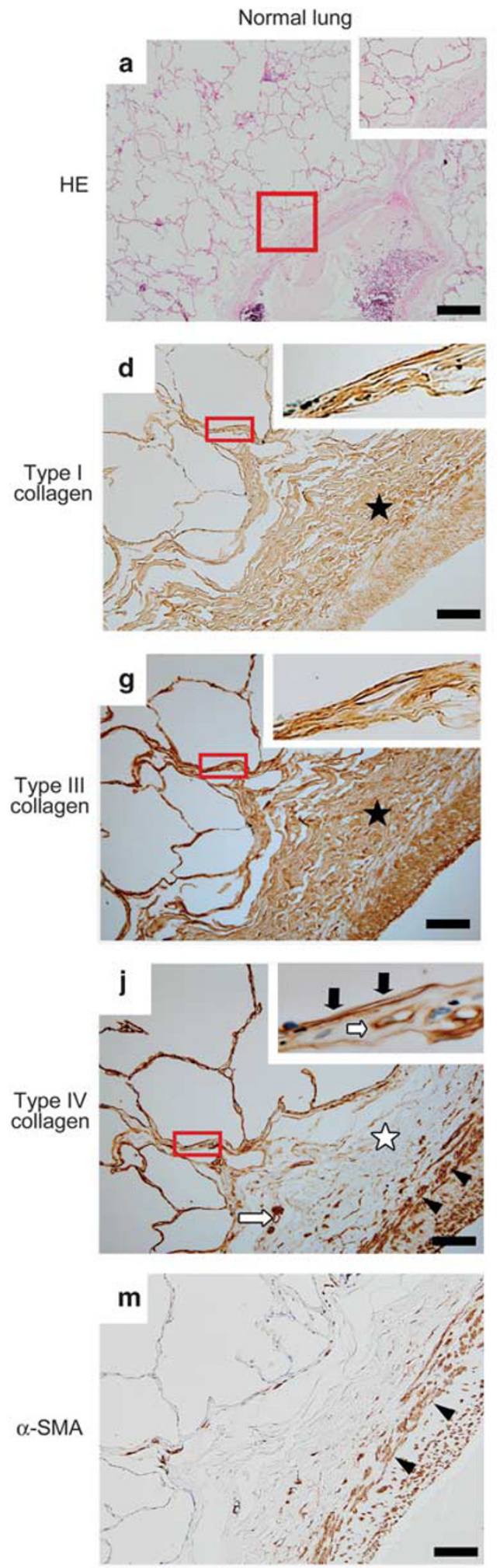
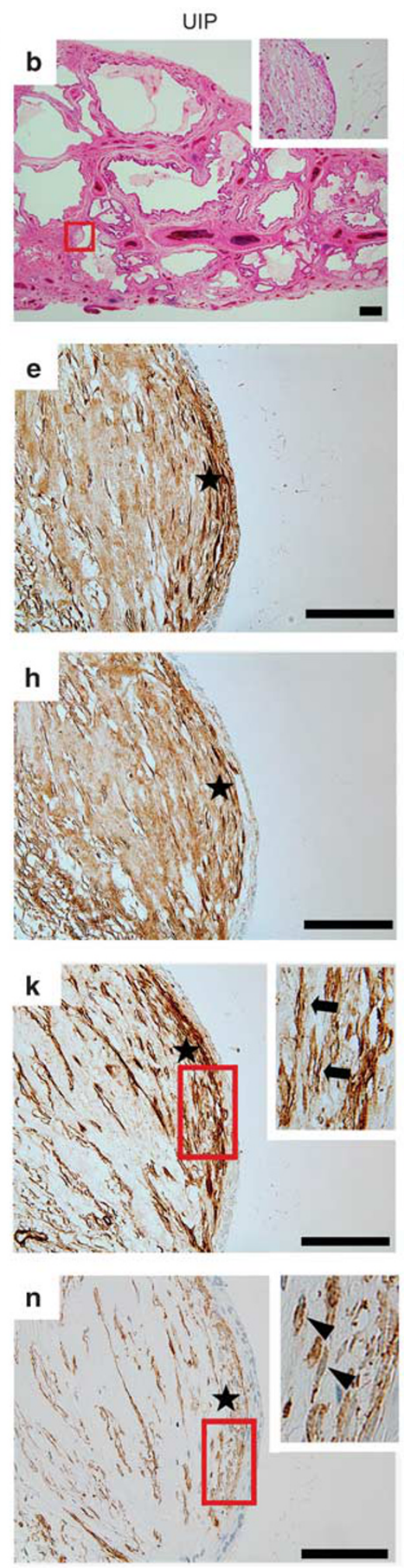
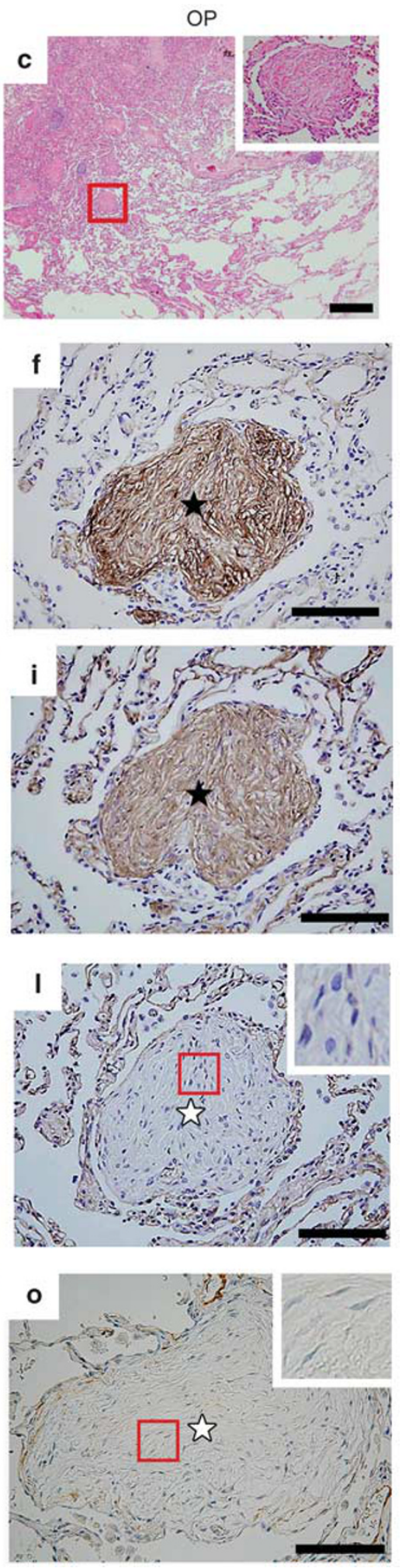
of $\alpha 1(\mathrm{IV})$ to $\alpha 6(\mathrm{IV})$ chains to mRNA expression of GAPDH in the same sample were determined relative to the ratios for cells cultured without TGF- $\beta 1$ in the same experiments.

\section{Reverse Transfection of siRNA Against a1(IV) and a2(IV) Chains}

Knockdown of $\alpha 1$ (IV) and $\alpha 2$ (IV) chains by reverse transfection of small interfering RNA (siRNA) was performed on day 1 after passage. siRNAs (SASI_Hs01_00018124 against $\alpha 1$ (IV) chain and SASI_Hs01_00116270 against $\alpha 2$ (IV) chain) were obtained from Sigma Aldrich Japan (Tokyo, Japan). The medium for reverse transfection consisted of $8 \mu$ l of MISSION siRNA against $\alpha 1(\mathrm{IV})$ and $\alpha 2(\mathrm{IV})$ chains, $16 \mu \mathrm{l}$ of Lipofectamine RNAiMAX (Invitrogen), $1.6 \mathrm{ml}$ of Opti-MEM I Medium (Invitrogen), and $6.4 \mathrm{ml}$ of DMEM with or without FBS and was used for reverse transfection according to the manufacturer's instructions. After $48 \mathrm{~h}$ of treatment, cells were used for migration, migration inhibition, and phosphorylation assays. To confirm the production of $\alpha 1$ (IV) and $\alpha 2$ (IV) chains, western blotting studies were also performed 3 days after reverse transfection with culture medium without FBS.

\section{Proliferation and Migration Assay}

To assess the effects of knockdown of $\alpha 1$ (IV) and $\alpha 2$ (IV) chains on fibroblast proliferation, $1.0 \times 10^{3}$ TIG-1-20 cells in 96-well plates were seeded and treated with $\alpha 1$ (IV) and $\alpha 2$ (IV) chain-specific siRNA or negative control treatment. Three days after these siRNA treatments, cell proliferation was assessed by bromodeoxyuridine incorporation according to the manufacturer's instructions (Roche Diagnostics, Mannheim, Germany). To examine the effects of knockdown of $\alpha 1$ (IV) and $\alpha 2$ (IV) chains on fibroblast migration, siRNA-treated TIG-1-20 cells, at a concentration of $1.0 \times 10^{6}$ cells $/ \mathrm{ml}$, in $300 \mu \mathrm{l}$ of serum-free DMEM were placed in the inserts of the Boyden chamber having an 8- $\mu \mathrm{m}$-pore membrane (BD Biosciences, Durham, NC, USA). DMEM supplemented with $10 \%$ FBS was placed as a chemoattractant in the bottom wells of the chamber (see the Results section on fibroblast migration). To examine the chemoattractant activity of $\alpha 1$ (IV) and $\alpha 2$ (IV) chains themselves, TIG-1-20 cells after knockdown of $\alpha 1$ (IV) and $\alpha 2(\mathrm{IV})$ chains at a concentration of $1.0 \times 10^{5}$ cells $/ \mathrm{ml}$ in $300 \mu \mathrm{l}$ of serum-free DMEM were placed in the inserts of the Boyden chamber and serum-free culture medium obtained from TIG-1-20 cells treated with siRNA against both $\alpha 1$ (IV) and $\alpha 2(\mathrm{IV})$ chains (not including $\alpha 1$ (IV) and $\alpha 2$ (IV) chains) or control siRNA (including $\alpha 1(\mathrm{IV})$ and $\alpha 2$ (IV) chains) was placed as a chemoattractant in the bottom wells of the chamber. The chamber was incubated for $12 \mathrm{~h}$ at $37^{\circ} \mathrm{C}$ under humidified $5 \% \mathrm{CO}_{2} / 95 \%$ air, and cells on the upper surface of the $8-\mu \mathrm{m}$ pore membrane of the insert were removed by scraping. The membrane was fixed and stained with Diff-Quik (Sysmex, Hyogo, Japan). Migration was assessed by using a light microscope (IX71; Olympus, Tokyo, Japan) to count the number of cells in three random high-power fields on the bottom surface of the insert membrane.

\section{Migration Inhibition Assay}

To investigate the effect of $\alpha 1$ (IV) and $\alpha 2$ (IV) chains on migration of fibroblasts, TIG-1-20 cells treated with siRNA against $\alpha 1$ (IV) and $\alpha 2$ (IV) chains or control siRNA were placed at a concentration of $1.0 \times 10^{5}$ cells $/ \mathrm{ml}$ in the inserts of the Boyden chamber, and then serum-free medium obtained from cultures of TIG-1-20 cells treated with control siRNA or siRNA against both $\alpha 1(\mathrm{IV})$ and $\alpha 2(\mathrm{IV})$ chains (medium including or not including $\alpha 1(\mathrm{IV})$ and $\alpha 2(\mathrm{IV})$ chains, respectively) was added to the inserts. DMEM supplemented with $10 \%$ FBS was placed as a chemoattractant in the bottom wells of the chamber. Cell migration was assessed in the same manner as in the migration assay.

\section{FAK Phosphorylation Assay}

To examine the effect of $\alpha 1$ (IV) or $\alpha 2$ (IV) chains on FAK phosphorylation in TIG-1-20 cells, after $48 \mathrm{~h}$ of treatment with $\alpha 1(\mathrm{IV})$ or $\alpha 2(\mathrm{IV})$ chain-specific siRNA or control siRNA, FBS was added to the treatment medium at a concentration of $0.5 \%$. At 30 min after adding FBS, cells were washed with PBS and lysed. Also, to investigate the replenishment effect of $\alpha 1$ (IV) and $\alpha 2$ (IV) chains on FAK phosphorylation, after $24 \mathrm{~h}$ of treatment with $\alpha$ (IV) and $\alpha 2$ (IV) chain-specific or control siRNA, siRNA treatment medium was replaced by serum-free culture medium obtained from TIG-1-20 cells treated with control siRNA or siRNA against both $\alpha 1(\mathrm{IV})$ and $\alpha 2$ (IV) chains (medium including or not including $\alpha 1(\mathrm{IV})$ and $\alpha 2$ (IV) chains, respectively), and then these siRNA-treated TIG-1-20 cells were cultured for $48 \mathrm{~h}$, washed with PBS, and lysed. Phosphorylated FAK and total FAK in TIG-1-20 cells were assessed by western blotting with antibodies against phosphorylated FAK (Tyr397) and total FAK at a dilution of 1:1000 (Cell Signaling Technology, Danvers, MA, USA). Immunoreactivity on blots was detected with the LAS-4000 Luminescent Image Analyzer with CCD Camera (Fujifilm).

\section{Statistical analysis}

Analysis of variance with Tukey comparisons was calculated by using StatView 5.0 (Hulinks, Tokyo, Japan). $P<0.05$ values were considered to be statistically significant.

\section{RESULTS \\ Deposition of Type I, III, and IV Collagens and a Chains of Type IV Collagen in Early Fibrotic Lesions of UIP and OP}

To investigate the expression and precise role of type IV collagen in IIPs, we used immunohistochemistry to study type IV collagen expression and compared it with the expression of type I and III collagens and $\alpha$-SMA by using tissue sections diagnosed as UIP and OP in addition to the normal lung (Figures 1a-c). Type I and III collagens were ubiquitously 
distributed in all interstitial components in the normal lung (Figures $1 \mathrm{~d}$ and $\mathrm{g}$, rectangular areas show alveolar septa and black stars indicate interstitium around pulmonary vessels). Type I and III collagens also demonstrated clear positive staining in all fibroblastic foci of UIP (Figures 1e and $\mathrm{h}$, black stars) and in intraluminal buds of OP (Figures if and $\mathrm{i}$, black stars) as well as in the normal lung interstitium (Figures 1d and g, black stars). Type IV collagen, however, was present only in the BM of normal lung (Figure 1j), i.e., in the $\mathrm{BM}$ of the epithelium (black arrows), endothelium (white arrows), and smooth muscle cells (arrowheads), but not in the interstitium around a pulmonary vessel (white star). However, in IIPs, in addition, type IV collagen's being present in the BM of normal lung area, it was clearly seen in fibroblastic foci of UIP (Figure 1k, black star) but not in intraluminal buds of OP (Figure 1l, white star). Fibroblasts in fibroblastic foci of UIP had strong positive staining for $\alpha$-SMA (Figure 1n, black star), in addition to type IV collagen staining, but fibroblasts in intraluminal buds of OP were only weakly positive or negative for $\alpha$-SMA (Figure 1o, white star). High-magnification views of fibroblastic foci (Figures $1 \mathrm{k}$ and $\mathrm{n}$, insets) showed that type IV collagen deposited in a fine fibrillar pattern (black arrows) around $\alpha$-SMA-positive fibroblasts (arrowheads), distinct from the BM structure.

We also studied the expression of all six $\alpha$ chains of type IV collagen in fibroblastic foci of UIP and intraluminal buds of OP. In normal lung, $\alpha 1$ (IV) and $\alpha 2$ (IV) chains were expressed in a continuous linear pattern in the BM of the epithelium (Figures 2a and d, black arrows), endothelium (white arrows), and smooth muscle cells (arrowheads). $\alpha 3$ (IV), $\alpha 4(\mathrm{IV}), \alpha 5$ (IV), and $\alpha 6$ (IV) chains, however, showed restricted expression: $\alpha 3$ (IV) and $\alpha 4(\mathrm{IV})$ chains were expressed only in the alveolar BM (Figure 2g, black arrows); the $\alpha 5$ (IV) chain was expressed in BMs of alveoli (Figure 2j, black arrows) and smooth muscle cells (arrowheads); and the $\alpha 6$ (IV) chain was expressed in BMs of bronchial epithelium and smooth muscle cells (data not shown), in agreement with earlier reports. ${ }^{18}$ Early fibrotic lesions of UIP and OP, however, had clear deposits of $\alpha 1$ (IV) and $\alpha 2(I V)$ chains only in fibroblastic foci of UIP (Figures $2 b$ and e, black stars), not in intraluminal buds of OP (Figures $2 \mathrm{c}$ and $\mathrm{f}$, white stars). $\alpha 1$ (IV) and $\alpha 2(\mathrm{IV})$ chains were present in a fine fibrillar pattern around fibroblasts (white arrows) in fibroblastic foci of UIP (Figure $2 \mathrm{~b}$ and e, insets). Deposition of $\alpha 3$ (IV) to $\alpha 6$ (IV) chains (data not shown for $\alpha 4$ (IV) and $\alpha 6$ (IV) chains) was not observed in early fibrotic lesions of UIP (Figures $2 \mathrm{~h}$ and $\mathrm{k}$ ) or of OP (Figures 2i and l). Each early fibrotic lesion in the same case had similar immunohistochemical features. Table 2 provides a summary of the immunohistochemical analysis of early fibrotic lesions of UIP and OP. All our data thus indicate that type IV collagen, especially $\alpha 1$ (IV) and $\alpha 2$ (IV) chains, was clearly deposited in fibroblastic foci, especially around $\alpha$-SMA-positive myofibroblasts in UIP but not in OP.

\section{Expression and Localization of a-SMA and Type IV Collagen in Cultured Lung Fibroblasts}

To investigate the role of $\alpha$ chains of type IV collagen in lung myofibroblasts in vitro (as we did in vivo), we studied the functional change of TIG-1-20 cells, a well-characterized cultured human lung fibroblast cell line, ${ }^{16}$ with TGF- $\beta 1$ stimulation or with the knockdown of $\alpha 1$ (IV) and $\alpha 2(\mathrm{IV})$ chains. Because TGF- $\beta 1$ stimulation has been reported to induce $\alpha$-SMA expression in fibroblasts as differentiation into myofibroblasts for 1 to 4 days, ${ }^{19-21}$ we cultured the cells for 0 to $168 \mathrm{~h}$ ( 7 days) with TGF- $\beta 1$ treatment to examine the effect of TGF- $\beta 1$ on mRNA expression of $\alpha$ (IV) chains in TIG-1-20 cells at various time points. We confirmed that the mRNA expression of $\alpha 1$ (IV) and $\alpha 2(\mathrm{IV})$ chains was upregulated in TIG-1-20 cells up to $96 \mathrm{~h}$ (4days; Supplementary Figure 2).

Then, we measured the expression of $\alpha(\mathrm{IV})$ chains in TIG-1-20 cells with various concentrations of TGF- $\beta 1$ at $72 \mathrm{~h}$, because we investigated the siRNA effect on the cells at $72 \mathrm{~h}$ after treatment according to the manufacturer's instructions. We found that $\alpha 1$ (IV) and $\alpha 2(I V)$ chains, but not $\alpha 3$ (IV) to $\alpha 6$ (IV) chains, were produced in the culture medium and that TGF- $\beta 1$ stimulation increased the production of $\alpha 1$ (IV) and $\alpha 2$ (IV) chains in a dose-dependent manner, similar to the production of $\alpha$-SMA by means of western blotting (Figures $3 a-d)$. We also confirmed that TGF- $\beta 1$ stimulation increased mRNA expression of $\alpha 1$ (IV) and $\alpha 2$ (IV) chains, but not of $\alpha 3$ (IV) to $\alpha 6$ (IV) chains, in a dose-dependent manner by RTqPCR (Figures 3e-g; data not shown for $\alpha 3$ (IV), $\alpha 4$ (IV), and $\alpha 6$ (IV) chains). Thus, we found that, in cultured lung fibroblasts in vitro, TGF- $\beta 1$ promoted myofibroblastic changes and production of type IV collagen, especially $\alpha 1$ (IV) and $\alpha 2$ (IV) chains, similar to the increased deposition of $\alpha 1(\mathrm{IV})$ and $\alpha 2$ (IV) chains around $\alpha$-SMA-positive myofibroblasts in early fibrotic lesions of UIP in vivo.

\section{Effects of a1(IV) and a2(IV) Chains on Migration of Cultured Lung Fibroblasts}

BMs provide structural support as well as regulating cell behavior, ${ }^{22}$ and several BM components, or even fragments of them, were found to regulate cell function. Arresten ${ }^{11}$ and canstatin, ${ }^{12}$ which are parts of the NC1 domains of $\alpha 1$ (IV) and $\alpha 2$ (IV) chains, respectively, inhibit endothelial cell migration. However, little is known about the function of $\alpha 1$ (IV) and $\alpha 2$ (IV) chains in the migration of lung fibroblasts. We therefore conducted a migration assay with TIG-1-20 cells after the knockdown of $\alpha 1(\mathrm{IV})$ and $\alpha 2(\mathrm{IV})$ chains. We obtained siRNA against $\alpha 1$ (IV) and $\alpha 2$ (IV) chains in TIG-1-20 cells treated with $1.0 \mathrm{ng} / \mathrm{ml}$ TGF- $\beta 1$ (Figures $4 \mathrm{a}-\mathrm{c}$ ) and found no difference in proliferation of TIG-1-20 cells treated with these siRNAs (Figure 4d). In contrast to the effect on cell proliferation, these siRNAs increased cell migration, as evaluated in a Boyden chamber assay with FBS as a chemoattractant, compared with migration of cells treated with control siRNA (Figure 4e-i). To examine whether $\alpha 1$ (IV) and $\alpha 2$ (IV) chains themselves acted as a 
Normal
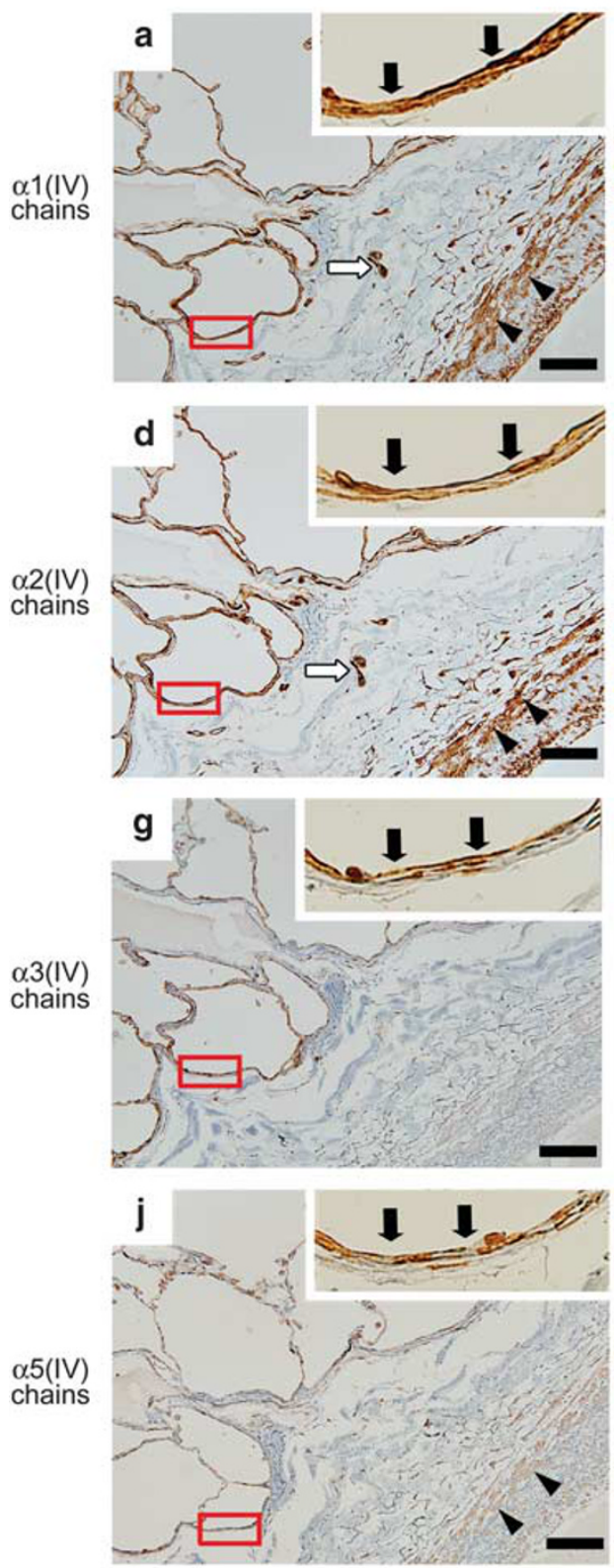

UIP
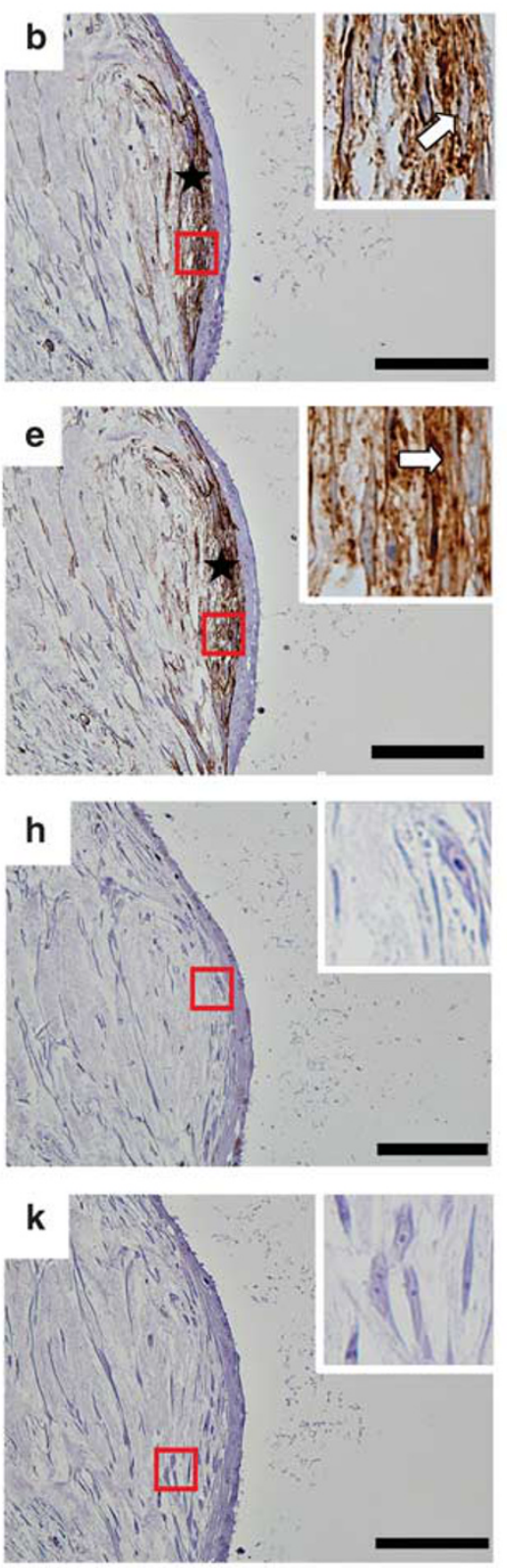

OP
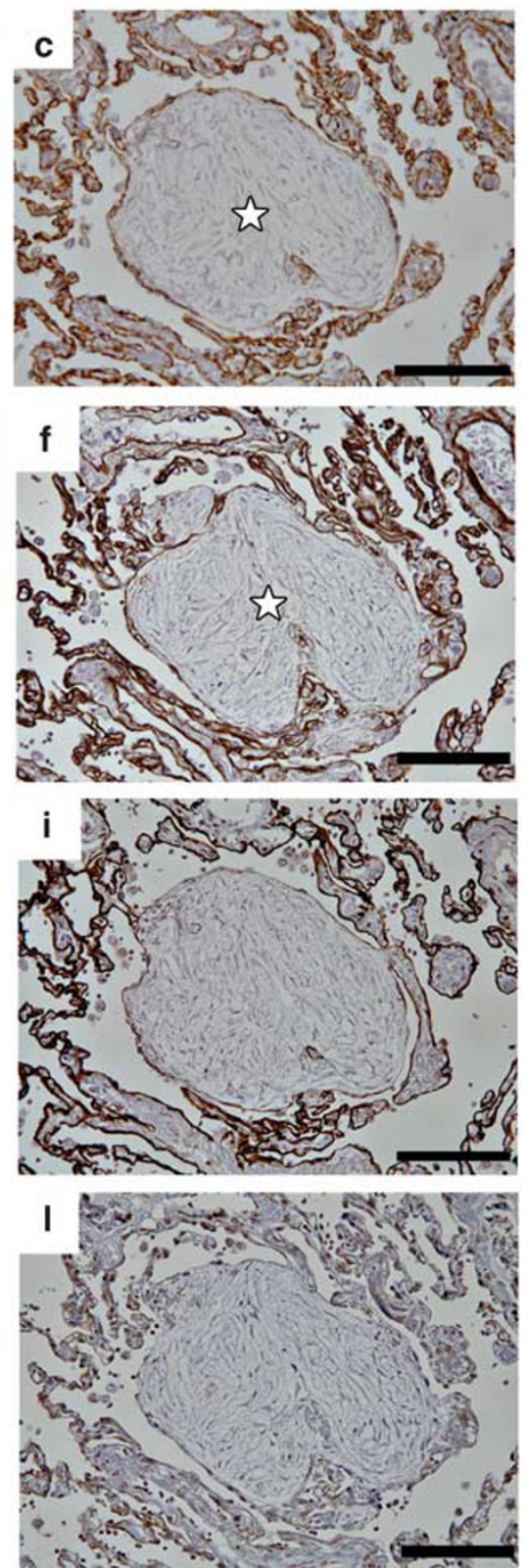

Figure 2 a Chains of type IV collagen (a(IV) chains) in normal control lung and early fibrotic lesions of usual interstitial pneumonia (UIP) and organizing pneumonia (OP). In normal control lung, a1(IV) (a) and a2(IV) (d) chains were widespread, in a continuous linear pattern, in the basement membrane (BM) of the alveolar epithelium (black arrows), endothelium (white arrows), and smooth muscle cells (arrowheads). The a3(IV) chain (g) was expressed only in the alveolar BM (black arrows), and the a5(IV) chain (j) was seen in BMs of alveoli (black arrows) and smooth muscle cells (arrowheads). In idiopathic interstitial pneumonias (IIPs), deposits of a1(IV) and a2(IV) chains, in addition to occurring in the BM, were clearly seen in the early fibrotic lesions of UIP ( $\mathbf{b}$ and $\mathbf{e}$, black stars) but not in those of OP (c and $\mathbf{f}$, white stars). a1(IV) and a2(IV) chains were present in a fine fibrillar pattern around fibroblasts (white arrows) in fibroblastic foci of UIP (b and $\mathbf{e}$, insets). Deposition of a3(IV) and a5(IV) chains was not seen in early fibrotic lesions of UIP ( $\mathbf{h}$ and $\mathbf{k}$ ) or of OP (i and $\mathbf{I}$ ). Insets show high-magnification views of the red rectangular areas. Images of normal control lung (a, $\mathbf{d}, \mathbf{g}$ and $\mathbf{j})$ were from the same patient, as were UIP images (b, e, $\mathbf{h}$ and $\mathbf{k}$ ) and OP images (c, $\mathbf{f}, \mathbf{i}$ and $\mathbf{I})$. Scale bars are $100 \mu \mathrm{m}$.

chemoattractant, we examined the chemoattractant activity of $\alpha 1$ (IV) and $\alpha 2$ (IV) chains and found that migration of TIG-120 cells treated with siRNA against $\alpha 1$ (IV) and $\alpha 2$ (IV) chains was not affected by placing culture medium with or without $\alpha 1(\mathrm{IV})$ and $\alpha 2$ (IV) chains as a chemoattractant in the bottom wells of the chamber (Figures $4 j$ and $k$ ). In contrast, adding control siRNA-treated culture medium, which included $\alpha 1$ (IV) and $\alpha 2(\mathrm{IV})$ chains produced by the control siRNAtreated cells, to the Boyden chamber inserts inhibited increased migration of cells treated with siRNA against both 
Table 2 Summary of immunoreactivity in early fibrotic lesions of UIP and OP

\begin{tabular}{lcccccccc}
\hline & \multicolumn{3}{c}{$\begin{array}{c}\text { Numbers of UIP } \\
\text { samples }(n=14)\end{array}$} & & \multicolumn{3}{c}{$\begin{array}{c}\text { Numbers of OP } \\
\text { samples }(n=11)\end{array}$} \\
\cline { 2 - 3 } Immunoreactivity & Strong & Weak & Negative & Strong & Weak & Negative \\
\hline a-SMA & 12 & 2 & 0 & & 4 & 6 \\
Type I collagen & 14 & 0 & 0 & & 11 & 0 & 0 \\
Type III collagen & 13 & 1 & 0 & & 11 & 0 & 0 \\
Type IV collagen & 13 & 1 & 0 & & 0 & 1 & 10 \\
a1(IV) chain & 12 & 2 & 0 & & 0 & 1 & 10 \\
a2(IV) chain & 12 & 2 & 0 & & 0 & 1 & 10 \\
a3-a6(IV) chains & 0 & 0 & 14 & & 0 & 0 & 11 \\
\hline
\end{tabular}

$\alpha 1($ IV) and $\alpha 2$ (IV) chains (Figures 41-o). Migration of TIG-120 cells treated with control siRNA, however, was not affected by the replacement of culture medium with or without $\alpha 1$ (IV) and $\alpha 2$ (IV) chains in the inserts of the chamber (Figures $4 p-r)$. Thus, our results showed that the knockdown of $\alpha 1$ (IV) and $\alpha 2$ (IV) chains enhanced the migration of lung fibroblasts and that $\alpha 1$ (IV) and $\alpha 2$ (IV) chains that had been produced by fibroblasts inhibited migration of fibroblasts, just as arresten ${ }^{11}$ and canstatin ${ }^{12}$ inhibited the migration of endothelial cells.

\section{FAK Phosphorylation in Cultured Lung Fibroblasts and Fibroblasts in Early Fibrotic Lesions of UIP and OP}

FAK activation promoted cell migration that was induced by integrin-extracellular matrix interactions. ${ }^{23}$ Arresten $^{24}$ and canstatin ${ }^{25,26}$ reportedly inhibited the phosphorylation of FAK, which resulted in the inhibition of endothelial cell migration. Therefore, we investigated the phosphorylation levels of FAK in TIG-1-20 cells after the knockdown of $\alpha$ (IV) and $\alpha 2$ (IV) chains. We found that these siRNAs increased the phosphorylation levels of FAK in TIG-1-20 cells compared with cells treated with control siRNA (Figures 5a-c). Furthermore, replacement of $\alpha 1(\mathrm{IV})$ and $\alpha 2$ (IV) chainspecific siRNA-treated medium (not including $\alpha 1$ (IV) and $\alpha 2$ (IV) chains) with control siRNA-treated medium (including $\alpha 1$ (IV) and $\alpha 2$ (IV) chains) reduced the increased phosphorylation levels of FAK in TIG-1-20 cells treated with siRNA against $\alpha 1$ (IV) and $\alpha 2$ (IV) chains (Figures $5 \mathrm{~d}$ and e). In contrast, replacement of control siRNA-treated medium with $\alpha 1$ (IV) and $\alpha 2(\mathrm{IV})$ chain-specific siRNA-treated medium did not change phosphorylation levels of FAK in TIG-120 cells treated with control siRNA (Figures $5 \mathrm{~d}$ and $\mathrm{f}$ ). We also investigated phosphorylated FAK in early fibrotic lesions of UIP and OP and found that fibroblasts in intraluminal buds of OP without $\alpha 1$ (IV) and $\alpha 2$ (IV) chains were positive for phosphorylated FAK (Figures 5i, 1 and o), but fibroblasts in fibroblastic foci of UIP with $\alpha 1$ (IV) and $\alpha 2$ (IV) chains were not (Figures $5 \mathrm{~h}, \mathrm{k}$ and $\mathrm{n}$ ). We found the following results for a

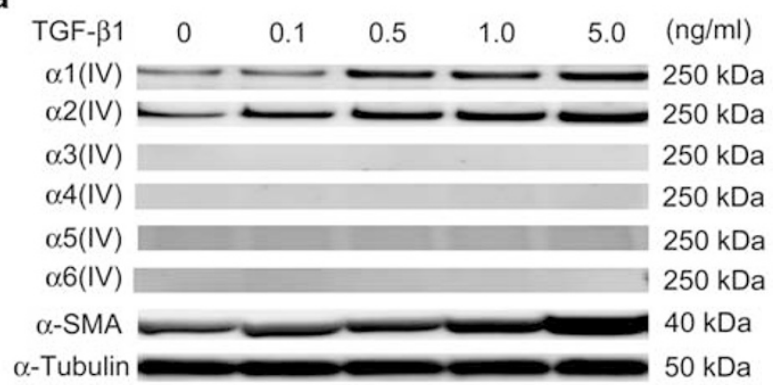

b
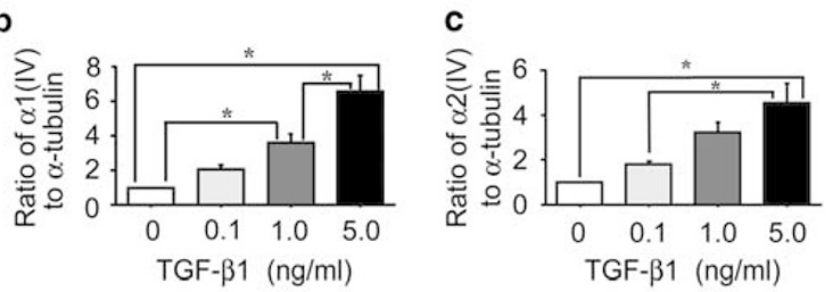

d
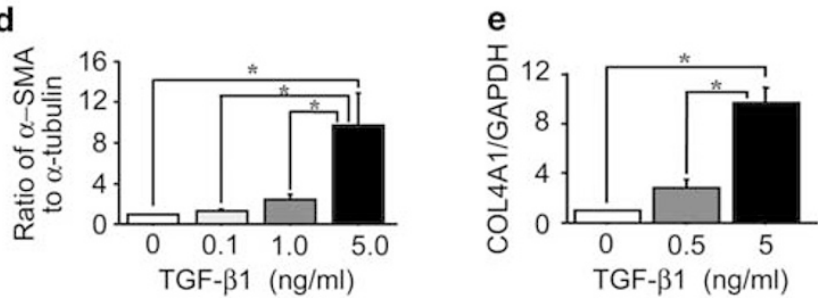

f
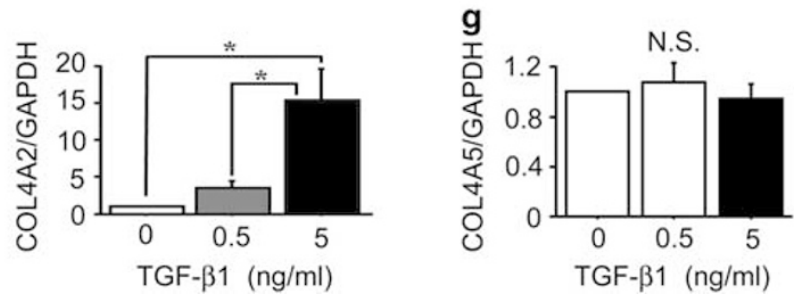

Figure 3 Dose-dependent upregulation of a chains of type IV collagen (a(IV) chains) - a1(IV) and a2(IV) chains-by transforming growth factor (TGF)- $\beta$ 1. (a) Representative western blots showing the amounts of a chains of type IV collagen in culture medium and the amounts of a-smooth muscle actin (a-SMA) and a-tubulin in TIG-1-20 cell lysates after treatment with different concentrations of TGF- $\beta 1$. Ratios of a1(IV) chain (b), a2(IV) chain (c), and a-SMA (d) to a-tubulin in the same sample were expressed relative to results for cells cultured without TGF- $\beta 1(=1.0)$ in the same experiments. Data from each experiment were quantified by means of densitometry. Ratios of mRNA expressions of a1(IV) (e, COL4A1), a2(IV) (f, COL4A2), or a5(IV) chains (g, COL4A5) to GAPDH in the same sample were analyzed via real-time quantitative reverse transcription-PCR (RT-qPCR) and expressed relative to results for cells cultured without TGF$\beta 1(=1.0)$ in the same experiments. Data are means \pm s.e.m. from three experiments. ${ }^{*} P<0.05$ for differences between indicated means. NS, not significant.

phosphorylated FAK immunoreactivity in early fibrotic lesions of UIP and OP: all 14 UIP lesions were negative for immunoreactivity, whereas of 11 OP lesions, 7 were positive and 4 were negative. Thus, our results suggested that $\alpha 1$ (IV) and $\alpha 2(I V)$ chains affected fibroblast migration in early fibrotic lesions of IIPs as well as in vitro, in part by changing the phosphorylation levels of FAK. 
a

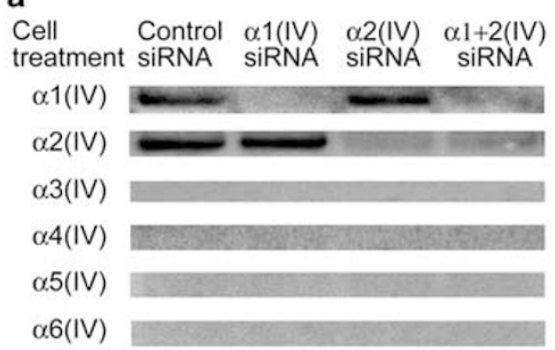

b

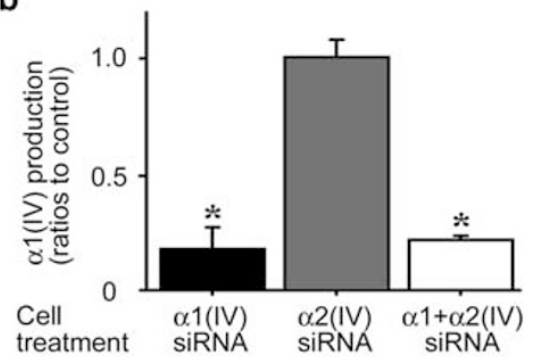

C

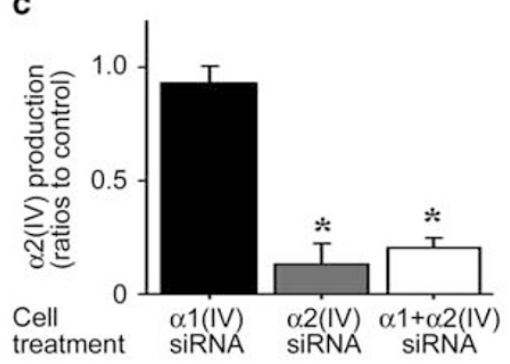

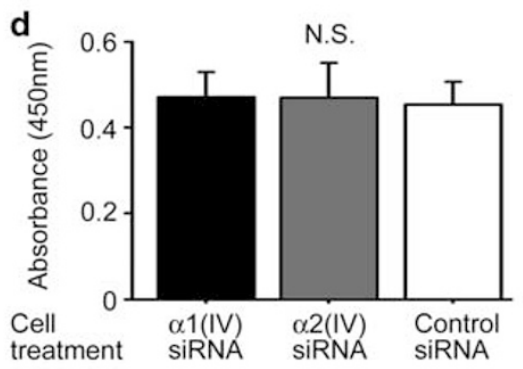

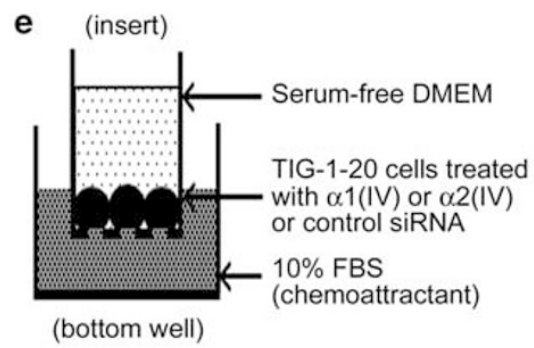

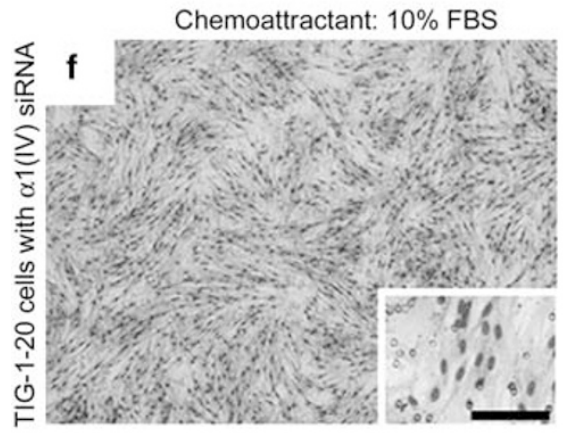

i

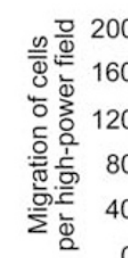

Cell

treatment

Chemo-

attractant

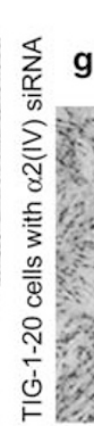

Chemoattractant: 10\% FBS

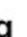

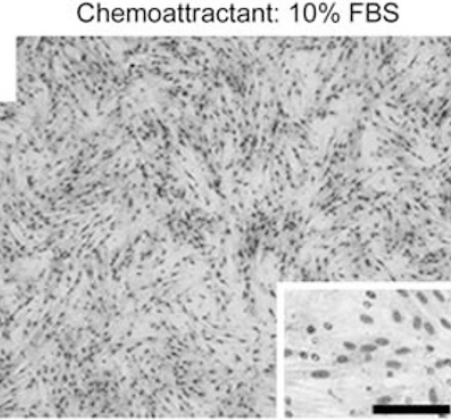

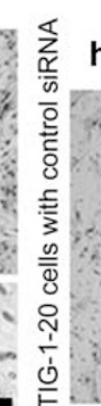

Chemoattractant: 10\% FBS

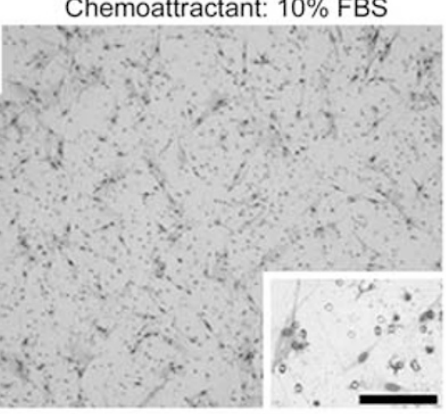

j (insert)

$*$

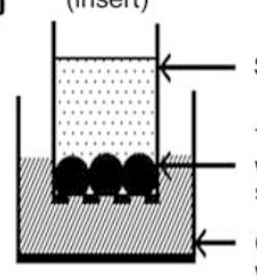

(bottom well)

Serum-free DMEM

TIG-1-20 cells treated

with $\alpha .1$ (IV) $+\alpha .2$ (IV)

SiRNA

Culture medium with or without $\alpha .1(\mathrm{IV})+\alpha .2$ (IV) chains (chemoattractant)

Figure 4 Effects of a chains of type IV collagen (a(IV) chains)—a1(IV) and a2(IV) chains—on migration of TIG-1-20 cells. (a) Representative western blots showing the production of a chains of type IV collagen by TIG-1-20 cells treated with small interfering RNA (siRNA) against a1 (IV) or a2 (IV) or a1 (IV) and a2 (IV) chains or control siRNA. Ratios of a1 (IV) chain (b) and a2(IV) chain (c) to a-tubulin in the same sample were expressed relative to the results for cells treated with control siRNA $(=1.0)$ in the same experiments. Data from each experiment were quantified by means of densitometry. (d) Proliferation of TIG-120 cells treated with siRNA against a1 (IV) chain or a2(IV) chain or control siRNA was determined by enzyme-linked immunosorbent assay, which measured the incorporation of 5-bromo-2'-deoxyuridine into DNA. (e) The design of the Boyden chamber assay for migration of TIG-1-20 cells treated with siRNA against a1(IV) or a2(IV) chains or control siRNA. (f-h) Representative images of migrating cells treated with siRNA against the a1 (IV) chain (f), a2(IV) chain (g), or control siRNA (h) with 10\% fetal bovine serum (FBS) as a chemoattractant. The insets show magnified views. (i) Numbers of migrating cells in three random high-power fields were counted. (j) The design of the Boyden chamber assay for chemoattractant activity of a1 (IV) and a2 (IV) chains. (k) Migration of TIG-1-20 cells after knockdown of a1(IV) and a2(IV) chains was also analyzed by using culture medium with or without a1 (IV) and a2(IV) chains as a chemoattractant instead of $10 \%$ FBS in the bottom wells of the chamber, and the numbers of migrating cells in three random high-power fields were counted. (I) The design of the Boyden chamber assay for the inhibitory effects of a1 (IV) and a2(IV) chains on fibroblast migration by exchanging medium that included $a 1$ (IV) and a2(IV) chains or did not include these chains. Representative images of migrating cells treated with siRNA against both a1(IV) and a2(IV) chains ( $\mathbf{m}$ and $\mathbf{n}$ ) or cells treated with control siRNA ( $\mathbf{p}$ and $\mathbf{q}$ ), with culture medium treated with siRNA against both a1(IV) and a2(IV) chains ( $\mathbf{m}$ and $\mathbf{p}$ ) or control siRNA ( $\mathbf{n}$ and $\mathbf{q}$ ) in the inserts of the chamber. Three random high-power fields were counted to obtain the number of migrating cells $\left(\mathbf{o}\right.$ and $\mathbf{r}$ ). Data are means \pm s.e.m. from three experiments. Scale bars are $100 \mu \mathrm{m}$. ${ }^{*} P<0.05$ for differences between indicated means. NS, not significant. 
$\mathbf{k}$

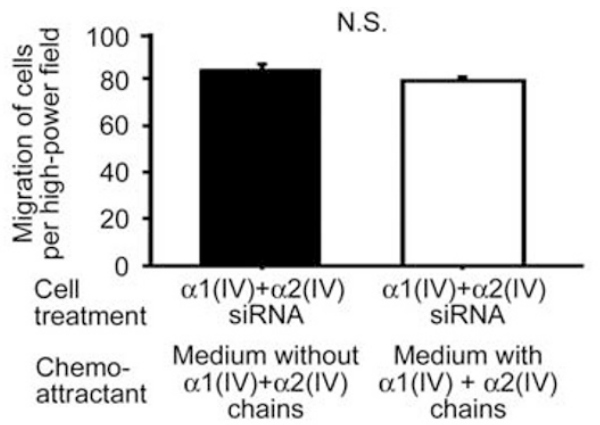

I

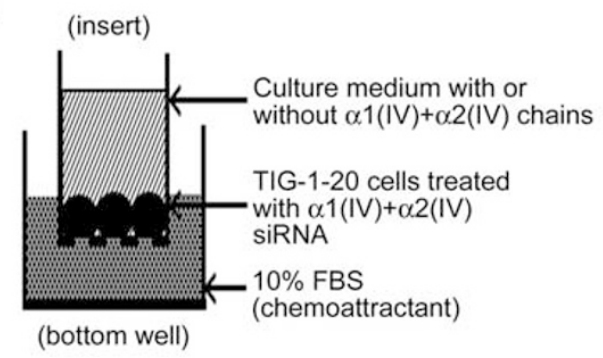

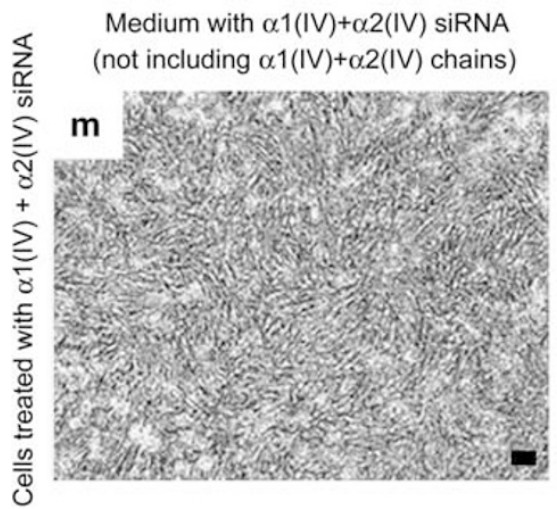

Medium with $\alpha 1(\mathrm{IV})+\alpha 2$ (IV) siRNA (not including $\alpha 1$ (IV) $+\alpha 2$ (IV) chains)

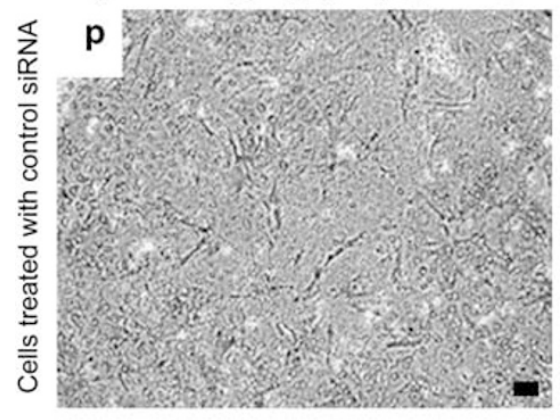

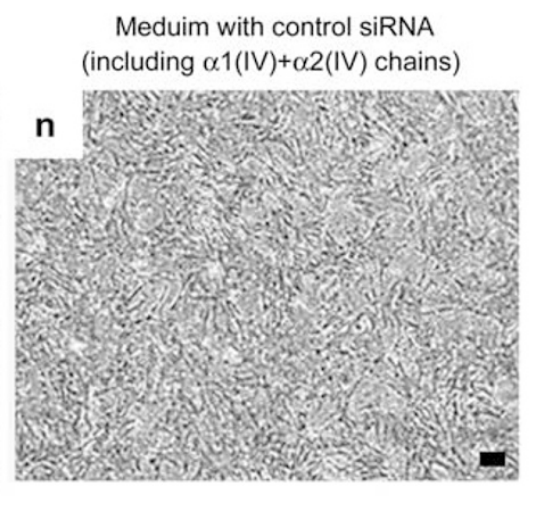

Meduim with control siRNA (including $\alpha .1$ (IV) $+\alpha .2$ (IV) chains)

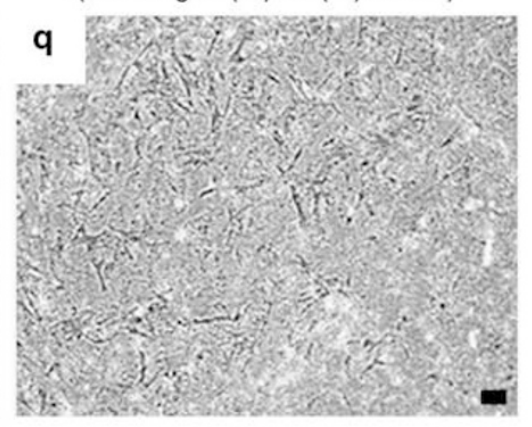

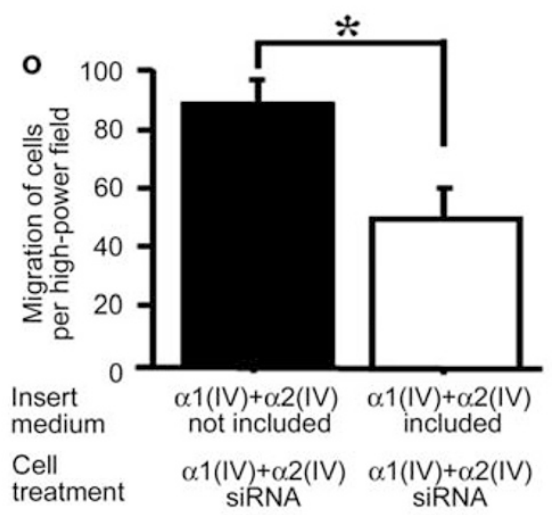

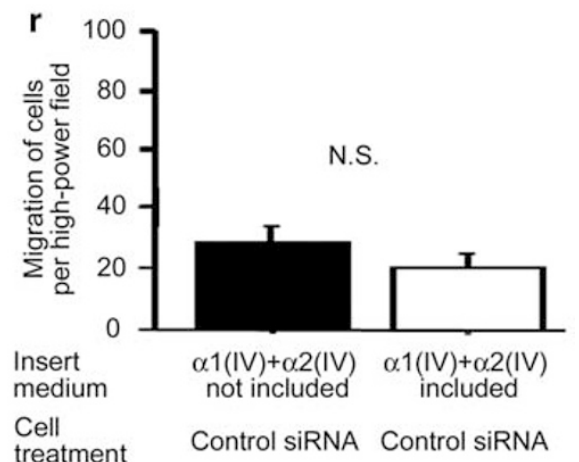

Figure 4 (Continued)

\section{DISCUSSION}

In the present study, we demonstrated, for the first time, the clear deposition of type IV collagen, especially $\alpha$ (IV) and $\alpha 2$ (IV) chains, but not $\alpha 3$ (IV) to $\alpha 6$ (IV) chains, in fibroblastic foci of UIP, which has a poor prognosis, but not in intraluminal buds of OP, which is an IIP with a good prognosis, with this result being similar to $\alpha$-SMA expression results in these lesions. Type I and III collagen deposits were, however, observed in early fibrotic lesions of both UIP and OP. TGF- $\beta 1$ enhanced myofibroblastic changes in cultured human lung fibroblasts, with increased production of $\alpha 1$ (IV) and $\alpha 2$ (IV) chains but not $\alpha 3$ (IV) to $\alpha 6$ (IV) chains in vitro. We also found that the knockdown of $\alpha 1$ (IV) and $\alpha 2$ (IV) chains enhanced the migration of lung fibroblasts and that $\alpha 1$
(IV) and $\alpha 2$ (IV) chains that had been produced by fibroblasts inhibited lung fibroblast migration. Furthermore, we showed that the knockdown of $\alpha 1$ (IV) and $\alpha 2$ (IV) chains increased the phosphorylation levels of FAK, and that adding $\alpha 1$ (IV) and $\alpha 2(I V)$ chains inhibited the increased phosphorylation levels of FAK in cultured lung fibroblasts. Clear positive findings for phosphorylated FAK in fibroblasts were observed in intraluminal buds of OP but not in fibroblastic foci of UIP. Therefore, deposition of type IV collagen in early fibrotic lesions, especially $\alpha 1$ (IV) and $\alpha 2$ (IV) chains, is involved in refractory pathophysiology of the IIPs including migration of lesion fibroblasts via a FAK pathway.

Type IV collagen, usually the most abundant constituent of the $\mathrm{BM}$, is also called the network-forming collagen because it 
can assemble into sheetlike networks. This network-forming property makes type IV collagen different from fibrillar collagens such as type I and III collagens, which have long ropelike structures and assemble into polymers called collagen fibrils. ${ }^{27}$ Although type IV collagen is normally present only in the BM, we found that it was deposited in fibroblastic foci of UIP in a fine fibrillar pattern. These results agree with reports of type IV collagen deposition in certain pathologic fibrotic conditions such as cirrhotic livers, in the perisinusoidal area and fibrous septa; ${ }^{28,29}$ diabetic nephropathy, in the mesangial matrix; ${ }^{30}$ and tumor-related fibrotic lesions, in a fibrillar pattern but not in the BM. ${ }^{31}$

We also found that type IV collagen was deposited around $\alpha$-SMA-positive myofibroblasts in fibroblastic foci of UIP but not in the intraluminal buds of OP, and that TGF- $\beta 1$ promoted the production of type IV collagen by cultured human lung fibroblasts together with increased expression of $\alpha$-SMA in the fibroblasts. In contrast, type I and III collagens were clearly deposited in not only fibroblastic foci of UIP but also intraluminal buds of OP, which had fewer $\alpha$-SMApositive myofibroblasts than UIP. In view of these results, we suggest that, compared with deposition of type I or III collagen, deposition of type IV collagen in early fibrotic lesions is more strongly correlated with myofibroblastic changes of fibroblasts, which are active in extracellular matrix production, in lesions of interstitial pneumonias with a poor prognosis.

Type IV collagen comprises six genetically distinct $\alpha$-chains designated $\alpha 1$ (IV) to $\alpha 6$ (IV) chains, and these chains interact and assemble with distinct specificity to form only three heterotrimers- $\alpha 1 \alpha 1 \alpha 2, \alpha 3 \alpha 4 \alpha 5$, and $\alpha 5 \alpha 5 \alpha 6$. $\alpha 1$ (IV) and $\alpha 2$ (IV) chains occur throughout the BMs of all tissues, whereas the other four chains have a restricted tissue distribution. For example, $\alpha 3$ (IV), $\alpha 4$ (IV), and $\alpha 5$ (IV) chains are present in the glomerular BM of the kidneys and alveolar BM of the lungs, whereas $\alpha 5$ (IV) and $\alpha 6$ (IV) chains are found in the BMs of smooth muscles and bronchial epithelium of the lungs. ${ }^{32} \mathrm{We}$ found that the increased deposits of type IV collagen in fibroblastic foci of UIP consisted of $\alpha$ (IV) and $\alpha 2$ (IV) chains but not $\alpha 3$ (IV) to $\alpha 6$ (IV) chains. Similarly, we found that TGF- $\beta 1$ stimulation, in a dose-dependent manner, increased the production and mRNA expression of $\alpha 1$ (IV) and $\alpha 2(\mathrm{IV})$ chains, but not $\alpha 3$ (IV) to $\alpha 6$ (IV) chains, by cultured human lung fibroblasts. Consistent with our data are findings that $\alpha 3$ (IV) to $\alpha 6$ (IV) chains tended to disappear in acinar and ductal BMs of the labial salivary gland in Sjögren's syndrome ${ }^{33}$ and in the BM of several malignant neoplasms, such as bronchioloalveolar carcinoma ${ }^{18}$ and gastric carcinoma, ${ }^{34}$ but that $\alpha 1$ (IV) and $\alpha 2$ (IV) chains were expressed continuously in the $\mathrm{BM}$ even in those pathologic conditions. Moreover, no reliable report about $\alpha 3$ (IV) to $\alpha 6$ (IV) chains being produced by cultured fibroblasts in vitro has been published. Our results together with these reports suggest that $\alpha 1$ (IV) and $\alpha 2$ (IV) chains, which differ from $\alpha 3$ (IV) to $\alpha 6$ (IV) chains, are a basic type of $\alpha$ chain of type IV collagen and may easily be produced by myofibroblasts in early fibrotic lesions of UIP, as when fibroblasts alone are stimulated by TGF- $\beta 1$ in vitro.

In addition to type IV collagen constituting the BM, type IV collagen has a molecular structure that reportedly contains a wealth of biochemical information. ${ }^{5,6}$ In type IV collagen, parts of NC1 domains of a single $\alpha(\mathrm{IV})$ chain-arresten $(\alpha 1$ chain) and canstatin ( $\alpha 2$ chain)-inhibited the phosphorylation of FAK by binding to integrins, which resulted in the inhibition of endothelial cell migration. ${ }^{24-26}$ We found that the knockdown of $\alpha 1$ (IV) and $\alpha 2$ (IV) chains increased cell migration with FAK phosphorylation in human lung fibroblasts and that $\alpha 1$ (IV) and $\alpha 2$ (IV) chains produced by lung fibroblasts reduced the increased levels of migration with FAK phosphorylation. Furthermore, clear positive findings for phosphorylated FAK were observed in fibroblasts in intraluminal buds of OP without $\alpha 1$ (IV) and $\alpha 2$ (IV) chain deposition, but not in fibroblastic foci of UIP with $\alpha$ (IV) and $\alpha 2$ (IV) chain deposition. Thus, we speculate that an increased deposition of type IV collagen, especially $\alpha$ (IV) and $\alpha 2$ (IV) chains, produced by lung fibroblasts in early fibrotic lesions of UIP inhibits the lesion fibroblast migration via inactivation of

Figure 5 Focal adhesion kinase (FAK) phosphorylation in cultured lung fibroblasts and fibroblasts in early fibrotic lesions of usual interstitial pneumonia (UIP) and organizing pneumonia (OP). (a) Representative western blots for phosphorylated FAK (pFAK) and total FAK in TIG-1-20 cell lysates treated with small interfering RNA (siRNA) against the a1 or a2 chain of type IV collagen (a1(IV) or a2(IV) chain) or control siRNA with or without adding fetal bovine serum (FBS). ( $\mathbf{b}$ and $\mathbf{c}$ ) Ratios of pFAK to total FAK in the same sample were expressed relative to results for cells cultured with control siRNA (=1.0) in the same sample after densitometric quantification. (d) Representative western blots for pFAK and total FAK in lysates of TIG-1-20 cells treated with siRNA against a1(IV) and a2(IV) chains or control siRNA were cultured with replacement medium obtained from cells treated with siRNA against both a1 (IV) and a2(IV) chains or control siRNA (medium not including or including a1(IV) and a2(IV) chains, respectively). (e and f) Ratios of pFAK to total FAK of cells treated with siRNA against a1(IV) and a2(IV) chains (e) or control siRNA (f) in the same sample were determined after densitometric quantification. Results were expressed as relative to the pFAK/total FAK ratios for cells cultured with replacement medium not including a1(IV) and a2(IV) chains (=1.0). Data are means \pm s.e.m. from three experiments. ${ }^{*} P<0.05$ for differences between indicated means. Representative hematoxylin-eosin-stained specimens of lung carcinoma as a positive control (g), UIP (h), and OP (i). Representative images of pFAK immunoreactivity in carcinomas (j), a fibroblastic focus (k), and intraluminal buds (I) corresponded to the red rectangular areas in (g-i), respectively. High-magnification views of carcinomas $(\mathbf{m})$, a fibroblastic focus (n), and intraluminal buds (o) were enlarged views of the red rectangular areas in (j-I), and insets showed higher-magnification views of the red rectangular areas in each image. Besides carcinoma cells as a positive control (j and $\mathbf{m}$ ), fibroblasts in intraluminal buds of OP (I and $\mathbf{o})$ showed pFAK-positive staining, especially in the cytoplasm (o inset), whereas fibroblasts in fibroblastic foci of UIP had no pFAK-positive staining ( $\mathbf{k}$ and $\mathbf{n})$. Images of lung carcinoma $(\mathbf{g}, \mathbf{j}$ and $\mathbf{m})$ were from the same patient, as were UIP images (h, $\mathbf{k}$ and $\mathbf{n})$ and OP images (i, I and $\mathbf{o})$. Scale bars are $1000 \mu \mathrm{m}(\mathbf{g}-\mathbf{i})$ and $100 \mu \mathrm{m}(\mathbf{j}-\mathbf{o})$. 
FAK, similar to the effect of arresten and canstatin on endothelial cells.

Recent findings suggest that FAK has a key role in the development of lung fibrosis including fibroblast migration. For example, FAK-deficient fibroblasts decrease their ability to migrate in vitro ${ }^{23}$, and the phosphorylation levels of FAK in fibroblasts are increased in a murine model of bleomycin- induced lung fibrosis. ${ }^{35}$ In general, an animal model of bleomycin-induced lung fibrosis is a fibrosis model with intraluminal buds of OP as a general process of repair from acute or subacute lung injury, ${ }^{36}$ but not a fibrosis model with fibroblastic foci of UIP as chronic, progressive and irreversible pulmonary damage with honeycomb change. ${ }^{37,38}$ In fact, to date, numerous agents have been shown to inhibit the fibrotic a
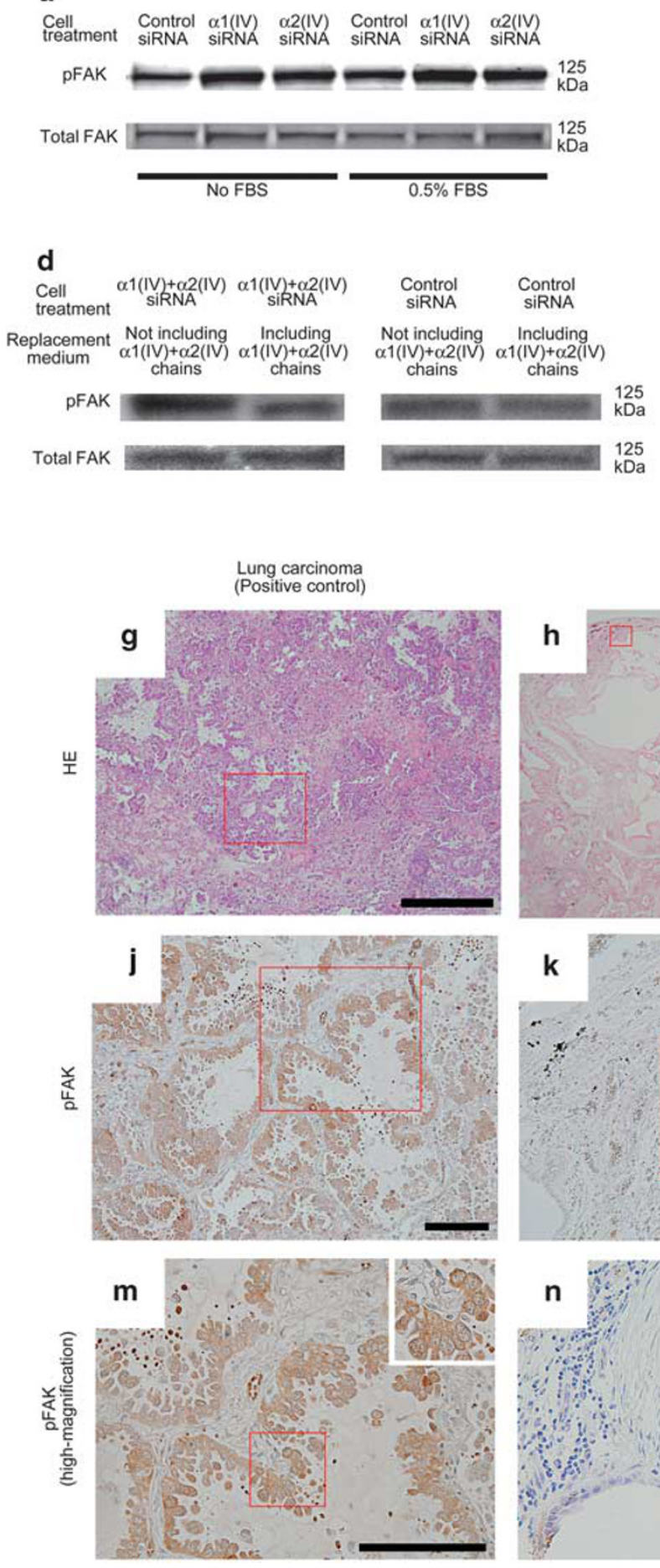

b No FBS

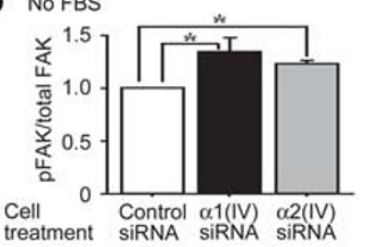

e

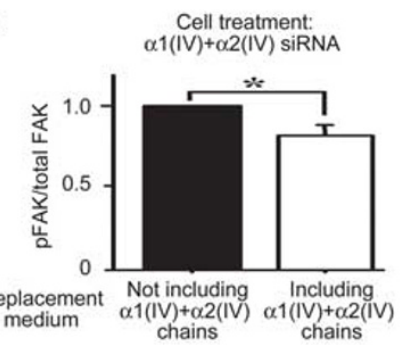

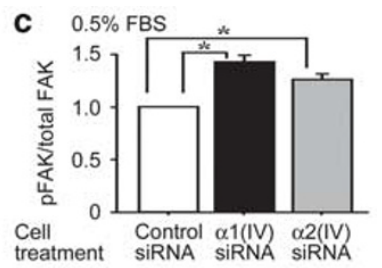

f

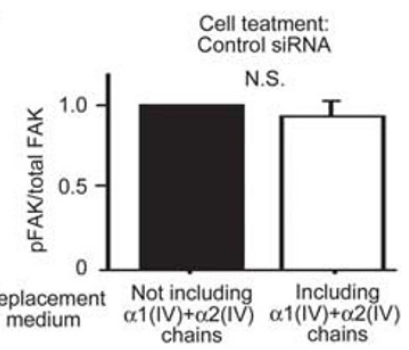

UIP
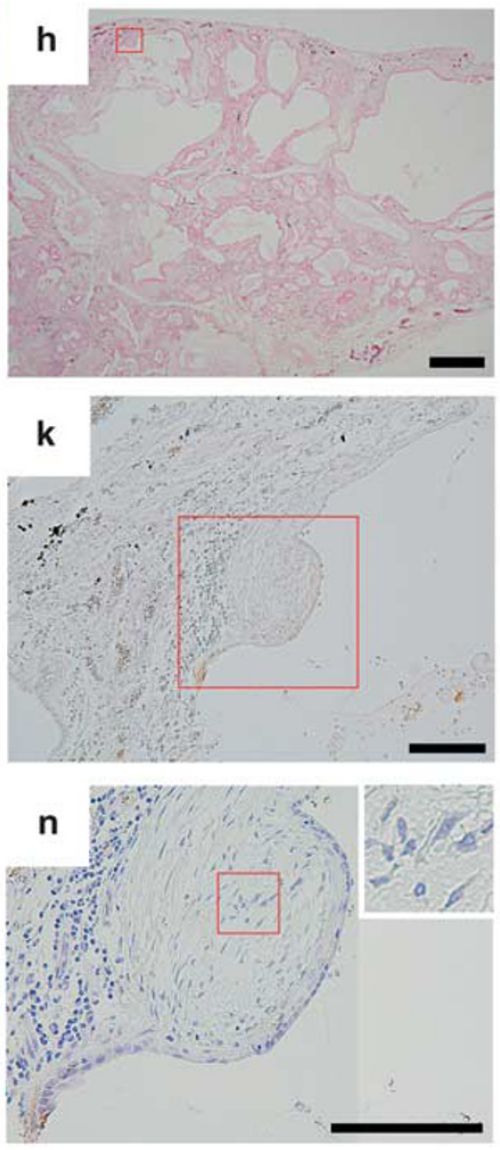

OP
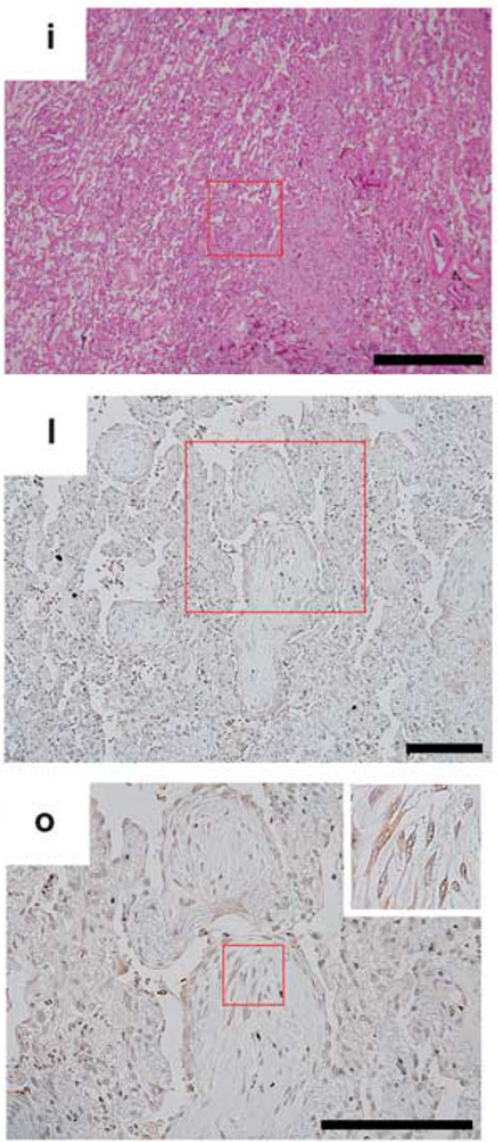
changes in this animal model, ${ }^{39}$ but a few of these agents, such as pirfenidone ${ }^{40,41}$ and nintedanib, ${ }^{42,43}$ have shown a comparable antifibrotic effect on cases of IPF/UIP in humans. The radiographic images of COP often demonstrate migratory consolidation corresponding to fibrotic lesions of OP, and thus the progression in fibrosis of COP is more acute (median, $<3$ months) $)^{1,2}$ than that of IPF which demonstrates a slow and gradual progression over many years. ${ }^{44}$ We have also previously reported that the deposition of matrix metalloproteinases, which function in extracellular matrix degradation and support the cell migration, ${ }^{45}$ is greater in intraluminal buds of OP than in fibroblastic foci of UIP. ${ }^{3}$ Taken together, these findings suggest that fibroblasts in intraluminal buds of $\mathrm{OP}$ have high migration activity compared with those in UIP. Therefore, our findings showing high levels of FAK phosphorylation in fibroblasts of OP are consistent with the idea that FAK has a key role in development of lung fibrosis.

To our knowledge, three studies (two immunohistochem$\mathrm{ical}^{46,47}$ and one western blotting ${ }^{48}$ studies) suggested that phosphorylated FAK levels were high in lung fibroblasts from IPF/UIP patients. However, in the three studies, the results were analyzed by comparison with normal human lung tissues or cultured lung fibroblasts from healthy subjects as normal controls, but not with intraluminal buds of $\mathrm{OP}$ as we examined in the present study. Therefore, the levels of phosphorylated FAK in fibroblasts in fibroblastic foci of UIP remains unclear compared with those in fibroblasts of early fibrotic lesions in other IIPs, especially in cases of COP.

In the present study, we first showed that lower levels of phosphorylated FAK in fibroblasts of fibroblastic foci in UIP compared with those of intraluminal buds in OP. It is known that the majority of fibrosis progression of UIP demonstrates a slow progression over many years. ${ }^{44}$ This is in accord with the studies which show that cultured fibroblasts from patients with IPF/UIP have low growth rate compared with that of normal controls ${ }^{49,50}$ and the proliferative activity of fibroblasts in fibroblastic foci of UIP tends to be lower than those in intraluminal buds of OP by Ki-67 immunoreactivity. ${ }^{51} \mathrm{We}$ also previously reported that deposition of tissue inhibitors of metalloproteinase-2, which inhibits cell migration, ${ }^{52}$ is greater in fibroblastic foci of UIP than in intraluminal buds of OP. ${ }^{3}$ Therefore, our results showing lower levels of FAK phosphorylation in fibroblasts of fibroblastic foci of UIP are in line with the characteristic features of UIP such as chronic and slowly progressive fibrosis, and thus may help to explain why the characteristic irreversible lung fibrosis of UIP is different from the reversible lung fibrosis as a general process of wound healing, such as COP or an animal model of bleomycininduced lung fibrosis, which both have fibroblasts with high levels of FAK phosphorylation.

Although the precise role of increased type IV collagen deposition in early fibrotic lesions of IIPs as related to the prognosis of each IIP remains unclear, it is interesting that especially $\alpha 1(\mathrm{IV})$ and $\alpha 2(\mathrm{IV})$ chains were produced by myofibroblasts and affected migration of fibroblasts in our in vitro studies, which corresponded to the results of our in vivo studies showing increased deposition of type IV collagen, especially $\alpha 1$ (IV) and $\alpha 2$ (IV) chains, in fibroblastic foci of UIP with a poor prognosis, but not in intraluminal buds of OP with a good prognosis. These results agree with the theory that interactions between cells and extracellular matrix, such as between fibroblasts and $\alpha 1$ (IV) and $\alpha 2$ (IV) chains in early fibrotic lesions of IIPs, are critical for the pathogenesis of lung fibrosis. ${ }^{53}$ Because we ${ }^{54}$ and El-Zammar et al. ${ }^{51}$ reported that some myofibroblasts in early fibrotic lesions were positive for Ki-67 in UIP and OP, we speculate that in early fibrotic lesions, some fibroblasts proliferate and are activated as myofibroblasts with abundant production of $\alpha 1$ (IV) and $\alpha 2$ (IV) chains and that $\alpha 1(\mathrm{IV})$ and $\alpha 2(\mathrm{IV})$ chains inhibit migration of lesion myofibroblasts, so that myofibroblasts tend to remain and continue to generate extracellular matrix in the lesions, thereby leading to refractory fibrosis in IIPs with a poor prognosis such as UIP.

In conclusion, the present study provides evidence that an increased deposition of $\alpha 1$ (IV) and $\alpha 2$ (IV) chains occurs around myofibroblasts in early fibrotic lesions of IIPs with a poor prognosis and that $\alpha 1(\mathrm{IV})$ and $\alpha 2(\mathrm{IV})$ chains that myofibroblasts produced may be implicated in the refractory pathophysiology of the IIPs, including migration of lesion fibroblasts. Continued investigation of type IV collagen in early fibrotic lesions of IIPs may contribute to understanding the pathogenesis of IIPs and thereby the treatment of IIPs.

Supplementary Information accompanies the paper on the Laboratory Investigation website (http://www.laboratoryinvestigation.org)

\section{ACKNOWLEDGMENTS}

We are grateful to Dr Yoshikazu Sado, director of immunological research at Shigei Medical Research Institute, for the generous donation of antibodies against a chains of type IV collagen, and Ms Judith B Gandy for her excellent editing of the manuscript. This work was supported in part by grants-in-aid for scientific research from the Ministry of Education, Culture, Sports, Science and Technology of Japan and in part by a grant from the Diffuse Lung Diseases Research Group from the Ministry of Health, Labor and Welfare of Japan.

\section{DISCLOSURE/CONFLICT OF INTEREST}

The authors declare no conflict of interest.

1. Society AT, Society ER. American Thoracic Society/European Respiratory Society International Multidisciplinary Consensus Classification of the Idiopathic Interstitial Pneumonias. This joint statement of the American Thoracic Society (ATS), and the European Respiratory Society (ERS) was adopted by the ATS board of directors, June 2001 and by the ERS Executive Committee, June 2001. Am J Respir Crit Care Med 2002;165:277-304.

2. Travis WD, Costabel U, Hansell DM et al. An official American Thoracic Society/European Respiratory Society statement: Update of the international multidisciplinary classification of the idiopathic interstitial pneumonias. Am J Respir Crit Care Med 2013;188:733-748.

3. Fukuda $\mathrm{Y}$, Ishizaki $\mathrm{M}$, Kudoh $\mathrm{S}$ et al. Localization of matrix metalloproteinases-1, -2 , and -9 and tissue inhibitor of metalloproteinase-2 in interstitial lung diseases. Lab Invest 1998;78:687-698.

4. Khalil N, O'Connor RN, Flanders KC et al. TGF-beta 1, but not TGF-beta 2 or TGF-beta 3 , is differentially present in epithelial cells of advanced 
pulmonary fibrosis: an immunohistochemical study. Am J Respir Cell Mol Biol 1996;14:131-138.

5. Timpl R. Structure and biological activity of basement membrane proteins. Eur J Biochem 1989;180:487-502.

6. Ingber $\mathrm{DE}$, Folkman J. How does extracellular matrix control capillary morphogenesis? Cell 1989;58:803-805.

7. O'Reilly MS, Boehm T, Shing $\mathrm{Y}$ et al. Endostatin: an endogenous inhibitor of angiogenesis and tumor growth. Cell 1997;88:277-285.

8. Dhanabal $M$, Ramchandran $R$, Volk $R$ et al. Endostatin: yeast production, mutants, and antitumor effect in renal cell carcinoma. Cancer Res 1999;59:189-197.

9. Timpl R, Brown JC. Supramolecular assembly of basement membranes. Bioessays 1996;18:123-132.

10. Prockop DJ, Kivirikko Kl. Collagens: molecular biology, diseases, and potentials for therapy. Annu Rev Biochem 1995;64:403-434.

11. Colorado PC, Torre A, Kamphaus $G$ et al. Anti-angiogenic cues from vascular basement membrane collagen. Cancer Res 2000;60:2520-2526.

12. Kamphaus GD, Colorado PC, Panka DJ et al. Canstatin, a novel matrixderived inhibitor of angiogenesis and tumor growth. J Biol Chem 2000;275:1209-1215.

13. Murawaki $Y$, Ikuta $Y$, Koda $M$ et al. Serum type III procollagen peptide, type IV collagen $7 \mathrm{~S}$ domain, central triple-helix of type IV collagen and tissue inhibitor of metalloproteinases in patients with chronic viral liver disease: relationship to liver histology. Hepatology 1994;20:780-787.

14. Tomino $Y$, Suzuki S, Azushima $C$ et al. Asian multicenter trials on urinary type IV collagen in patients with diabetic nephropathy. J Clin Lab Anal 2001:15:188-192.

15. Kasuga I, Yonemaru M, Kiyokawa $\mathrm{H}$ et al. Clinical evaluation of serum type IV collagen $7 \mathrm{~S}$ in idiopathic pulmonary fibrosis. Respirology 1996;1:277-281.

16. Ohashi M, Aizawa S, Ooka $\mathrm{H}$ et al. A new human diploid cell strain, TIG-1, for the research on cellular aging. Exp Gerontol 1980;15:121-133.

17. Sado $Y$, Kagawa $M$, Kishiro $Y$ et al. Establishment by the rat lymph node method of epitope-defined monoclonal antibodies recognizing the six different alpha chains of human type IV collagen. Histochem Cell Biol 1995;104:267-275.

18. Nakano KY, Iyama Kl, Mori T et al. Loss of alveolar basement membrane type IV collagen alpha3, alpha4, and alpha5 chains in bronchioloalveolar carcinoma of the lung. J Pathol 2001;194:420-427.

19. Hashimoto S, Gon Y, Takeshita I et al. Transforming growth Factorbeta1 induces phenotypic modulation of human lung fibroblasts to myofibroblast through a c-Jun-NH2-terminal kinase-dependent pathway. Am J Respir Crit Care Med 2001;163:152-157.

20. Chaudhary NI, Roth GJ, Hilberg F et al. Inhibition of PDGF, VEGF and FGF signalling attenuates fibrosis. Eur Respir J 2007;29:976-985.

21. Yokozeki M, Moriyama K, Shimokawa $\mathrm{H}$ et al. Transforming growth factor-beta 1 modulates myofibroblastic phenotype of rat palatal fibroblasts in vitro. Exp Cell Res 1997;231:328-336.

22. Kalluri R. Basement membranes: structure, assembly and role in tumour angiogenesis. Nat Rev Cancer 2003;3:422-433.

23. Sieg DJ, Hauck CR, Schlaepfer DD. Required role of focal adhesion kinase (FAK) for integrin-stimulated cell migration. J Cell Sci 1999;112: 2677-2691.

24. Sudhakar A, Nyberg P, Keshamouni VG et al. Human alpha1 type IV collagen NC1 domain exhibits distinct antiangiogenic activity mediated by alpha1beta1 integrin. J Clin Invest 2005;115:2801-2810.

25. Panka DJ, Mier JW. Canstatin inhibits Akt activation and induces Fasdependent apoptosis in endothelial cells. J Biol Chem 2003;278: 37632-37636

26. Magnon C, Galaup A, Mullan B et al. Canstatin acts on endothelial and tumor cells via mitochondrial damage initiated through interaction with alphavbeta3 and alphavbeta5 integrins. Cancer Res 2005;65:4353-4361.

27. van der Rest M, Garrone R. Collagen family of proteins. FASEB J 1991;5 2814-2823.

28. Hahn E, Wick G, Pencev D et al. Distribution of basement membrane proteins in normal and fibrotic human liver: collagen type IV, laminin, and fibronectin. Gut 1980;21:63-71.

29. Tsutsumi M, Urashima S, Matsuda $Y$ et al. Changes in type IV collagen content in livers of patients with alcoholic liver disease. Hepatology 1993;17:820-827.
30. Watanabe $\mathrm{H}$, Sanada $\mathrm{H}$, Shigetomi $\mathrm{S}$ et al. Urinary excretion of type IV collagen as a specific indicator of the progression of diabetic nephropathy. Nephron 2000;86:27-35.

31. Chen Y, Satoh T, Sasatomi E et al. Critical role of type IV collagens in the growth of bile duct carcinoma. In vivo and in vitro studies. Pathol Res Pract 2001;197:585-596.

32. Yoshioka K, Hino S, Takemura T et al. Type IV collagen alpha 5 chain. Normal distribution and abnormalities in X-linked Alport syndrome revealed by monoclonal antibody. Am J Pathol 1994;144:986-996.

33. Poduval $\mathrm{P}$, Sillat $\mathrm{T}$, Virtanen I et al. Abnormal basement membrane type IV collagen alpha-chain composition in labial salivary glands in Sjögren's syndrome. Arthritis Rheum 2009:60:938-945.

34. Baba $\mathrm{Y}$, lyama K, Ikeda $\mathrm{K}$ et al. Differential expression of basement membrane type IV collagen alpha chains in gastric intramucosal neoplastic lesions. J Gastroenterol 2007;42:874-880.

35. Vittal R, Horowitz JC, Moore BB et al. Modulation of prosurviva signaling in fibroblasts by a protein kinase inhibitor protects against fibrotic tissue injury. Am J Pathol 2005;166:367-375.

36. Adamson IY, Bowden DH. The pathogenesis of bleomycin-induced pulmonary fibrosis in mice. Am J Pathol 1974;77:185-197.

37. Izbicki G, Segel MJ, Christensen TG et al. Time course of bleomycininduced lung fibrosis. Int J Exp Pathol 2002;83:111-119.

38. Chua F, Gauldie J, Laurent GJ. Pulmonary fibrosis: searching for model answers. Am J Respir Cell Mol Biol 2005;33:9-13.

39. Moeller A, Ask K, Warburton D et al. The bleomycin animal model: a useful tool to investigate treatment options for idiopathic pulmonary fibrosis? Int J Biochem Cell Biol 2008;40:362-382.

40. King TE, Bradford $W Z$, Castro-Bernardini $S$ et al. A phase 3 trial of pirfenidone in patients with idiopathic pulmonary fibrosis. N Engl J Med 2014;370:2083-2092.

41. Schaefer CJ, Ruhrmund DW, Pan L et al. Antifibrotic activities of pirfenidone in animal models. Eur Respir Rev 2011;20:85-97.

42. Richeldi L, du Bois RM, Raghu $G$ et al. Efficacy and safety of nintedanib in idiopathic pulmonary fibrosis. N Engl J Med 2014;370:2071-2082.

43. Wollin L, Maillet I, Quesniaux V et al. Antifibrotic and anti-inflammatory activity of the tyrosine kinase inhibitor nintedanib in experimental models of lung fibrosis. J Pharmacol Exp Ther 2014;349:209-220.

44. Raghu G, Collard HR, Egan JJ et al. An official ATS/ERS/JRS/ALAT statement: idiopathic pulmonary fibrosis: evidence-based guidelines for diagnosis and management. Am J Respir Crit Care Med 2011;183:788-824.

45. Björklund $M$, Koivunen E. Gelatinase-mediated migration and invasion of cancer cells. Biochim Biophys Acta 2005:1755:37-69.

46. Ding $\mathrm{Q}, \mathrm{Cai} \mathrm{GQ}, \mathrm{Hu} \mathrm{M}$ et al. FAK-related nonkinase is a multifunctional negative regulator of pulmonary fibrosis. Am J Pathol 2013;182: 1572-1584.

47. Lagares D, Busnadiego O, García-Fernández RA et al. Inhibition of focal adhesion kinase prevents experimental lung fibrosis and myofibroblast formation. Arthritis Rheum 2012;64:1653-1664.

48. Cai GQ, Zheng A, Tang Q et al. Downregulation of FAK-related nonkinase mediates the migratory phenotype of human fibrotic lung fibroblasts. Exp Cell Res 2010;316:1600-1609.

49. Ramos C, Montaño M, García-Alvarez J et al. Fibroblasts from idiopathic pulmonary fibrosis and normal lungs differ in growth rate, apoptosis, and tissue inhibitor of metalloproteinases expression. Am J Respir Cell Mol Biol 2001:24:591-598.

50. Hetzel $M$, Bachem $M$, Anders $D$ et al. Different effects of growth factors on proliferation and matrix production of normal and fibrotic human lung fibroblasts. Lung 2005;183:225-237.

51. El-Zammar O, Rosenbaum P, Katzenstein AL. Proliferative activity in fibrosing lung diseases: a comparative study of Ki-67 immunoreactivity in diffuse alveolar damage, bronchiolitis obliterans-organizing pneumonia, and usual interstitial pneumonia. Hum Pathol 2009;40:1182-1188.

52. Brew K, Nagase $\mathrm{H}$. The tissue inhibitors of metalloproteinases (TIMPs) an ancient family with structural and functional diversity. Biochim Biophys Acta 2010;1803:55-71.

53. Paine R, Ward PA. Cell adhesion molecules and pulmonary fibrosis. Am J Med 1999;107:268-279.

54. Kang $\mathrm{D}$, Nakayama $\mathrm{T}$, Togashi $\mathrm{M}$ et al. Two forms of diffuse alveolar damage in the lungs of patients with acute respiratory distress syndrome. Hum Pathol 2009;40:1618-1627. 